Article

\title{
Elemental Geochemical Evidence for Depositional Conditions and Organic Matter Enrichment of Black Rock Series Strata in an Inter-Platform Basin: The Lower Carboniferous Datang Formation, Southern Guizhou, Southwest China
}

\author{
Jianghui Ding ${ }^{1,2,3}$, Jinchuan Zhang ${ }^{1,2}, *$, Xuan Tang ${ }^{1,2}$, Zhipeng Huo ${ }^{1,2}$, Shuangbiao Han ${ }^{4}$, \\ Yue Lang ${ }^{1,2}$, Yuyan Zheng ${ }^{1,2}$, Xingqi $\mathrm{Li}^{1,2}$ and Tong Liu ${ }^{1,2}$ \\ 1 School of Energy and Resources, China University of Geosciences, Beijing 100083, China; \\ djhdream2015@163.com (J.D.); tangxuan@cugb.edu.cn (X.T.); huozhipeng521@163.com (Z.H.); \\ gouqilier@126.com (Y.L.); 18801100712@163.com (Y.Z.); 3006180037@cugb.edu.cn (X.L.); \\ liutong9306@163.com (T.L.) \\ 2 Key Laboratory of Strategy Evaluation for Shale Gas, Ministry of Land and Resources, Beijing 100083, China \\ 3 Key Laboratory of Tectonics and Petroleum Resources, Ministry of Education, China University of \\ Geosciences, Wuhan 430074, China \\ 4 College of Geoscience and Surveying Engineering, China University of Mining and Technology, \\ Beijing 100083, China; bjcuphan@163.com \\ * Correspondence: zhangjc@cugb.edu.cn; Tel.: +86-10-8232-2735
}

Received: 7 September 2018; Accepted: 3 November 2018; Published: 6 November 2018

check for updates

\begin{abstract}
The Datang Formation black rock series deposited in an inter-platform basin are considered to be one of the main source rocks in Southwest China. However, their depositional conditions and organic matter enrichment in such a setting are not well understood. In this study, geochemical characteristics of total organic carbon (TOC), major, trace and rare earth elements (REEs) of thirty black rock series samples from a newly-cut roadside outcrop (Zhenning section) in Southern Guizhou, Southwest China are systematically investigated to examine provenance, paleoredox conditions, paleoclimate, paleoproductivity and sedimentary rate, and further to explore their influence on organic matter enrichment. Results show that TOC contents of the investigated samples range from $0.90 \%$ to $2.83 \%$, with an average of $1.45 \%$, with TOC variations being in accord with the stratigraphic cyclicity. These data also indicate that the target black rock series were deposited in a combination of continental island arc and active continental margin tectonic setting that exhibited moderate weathering, oxic/dysoxic oxygen levels, hot and arid to semiarid climate, and a higher sedimentary rate. Multiple paleoredox indicators (e.g., $\mathrm{Ni} / \mathrm{Co}, \mathrm{V} / \mathrm{Cr}, \mathrm{U} / \mathrm{Th}$ and $\mathrm{V} /(\mathrm{V}+\mathrm{Ni})$ ratios), together with slightly negative $\mathrm{Ce}$ anomalies and $\mathrm{EF}_{\mathrm{U}}-\mathrm{EF}_{\mathrm{Mo}}$ covariation, totally suggest an oxic/dysoxic water environment, which is usually considered unfavorable for organic matter preservation. Average $(\mathrm{La} / \mathrm{Yb})_{\mathrm{N}}$ ratios (1.41) reflect a higher sedimentary rate, resulting in shortening the exposure time of organic matter in the degradation region of aerobic bacteria under oxic/dysoxic conditions. Of the three factors that could affect organic matter enrichment, including paleoproductivity, paleoredox conditions and sedimentary rate, the relatively high paleoproductivity indicated by the relatively high TOC contents appears to be the dominant factor. In contrast, the other two factors, i.e., paleoredox conditions and the rate of sedimentation play relatively less significant roles. Based on these factors, an enrichment model of organic matter for the black rock series in Southern Guizhou Depression is established. These findings also contribute to constraining the sedimentary environment in such a setting and further deepen our understanding of the potential shale gas resources of the Southern Guizhou Province.
\end{abstract}


Keywords: elemental geochemistry; black rock series; depositional condition; organic matter enrichment; Datang Formation; inter-platform basin

\section{Introduction}

In the past few decades, increasing numbers of scholars have demonstrated the applicability of understanding major elements, trace elements (TEs), and rare earth elements (REEs, from La to Lu) data as useful indicators to examine the depositional conditions (e.g., provenance, paleoredox conditions and paleoclimate) [1-7] and to determine the mechanisms controlling the organic matter enrichment in modern and ancient sediments [8-11]. Various studies have covered the sedimentary environments including marine shale in intra-shelf basins, continental shale in lacustrine rift basins and transitional shale in residual bays [5,6,12-16]. However, exceedingly few publications document the organic-rich sediments deposited in an inter-platform basin owing to their limited occurrence in the stratigraphic records [17-19]. Fortunately, the Datang Formation black rock series in Southern Guizhou, Southwest China, provides an invaluable opportunity to characterize the inorganic geochemical characteristics and further to explore their depositional conditions and organic matter enrichment in such a setting.

Previous studies suggested that organic-rich black rock series from the Lower Carboniferous Datang Formation are considered to be deposited in an inter-platform basin $[18,20]$ and be the dominating hydrocarbon source rocks in Southwest China [21,22]. The sedimentary environment of the Datang Formation in Southern Guizhou Depression has been studied by means of lithologic description, petrographic and biostratigraphic approaches [18,20,23,24]. However, the above-mentioned methods for elucidating sedimentary environments are restricted by the number of well drillings. More importantly, few wells have been drilled in the studied region until now, so elemental geochemical characteristics of black rock series from outcrop can provide valuable information for systematically deciphering sedimentary environments. Additionally, elemental geochemical methods are employed for reconstructing the sedimentary environments more easily, reproducibly and efficiently $[6,25]$. The sediment provenance of the Lower Carboniferous is also investigated based on the detrital mineral composition analyses [26], but less attention is focused on the elemental geochemical approaches [27], despite the fact that elemental geochemical approaches can better characterize the provenance and paleoclimate fluctuations.

In this study, samples of black rock series collected from a newly-cut roadside outcrop in Zhenning county, Anshun city, Southwest China, were analyzed for total organic carbon (TOC), as well as major elements, TEs, and REEs concentrations. We aim to place a better understanding on: (1) the elemental geochemical characteristics of black rock series in an inter-platform basin; (2) the provenance, paleoredox conditions, paleoclimate, paleoproductivity and sedimentary rate at the time of the black rock series deposition; and (3) the roles of depositional conditions in organic matter enrichment in the Datang Formation.

\section{Geological Setting}

Located in the southern part of Guizhou Province, Southwest China (Figure 1a), the studied area tectonically belongs to Southern Guizhou Depression, southwestern edge of Upper Yangtze Plate $[20,27]$ and has experienced multi-stage complex tectonic movements and resulted in forming a series of folds with axes trending NW and strike faults with the NW- or NE-trending [21,27]. The strata exposed at the surface in this region are mainly Devonian, Carboniferous and Permian. During the early Carboniferous period, the regionally extensive black rock series developed on the Upper Yangtze Plate as well as its periphery, representing a large-scale transgression process [20,21]. A relatively shallow-water inter-platform basin formed in the foregoing depression in southern Guizhou [20]. 


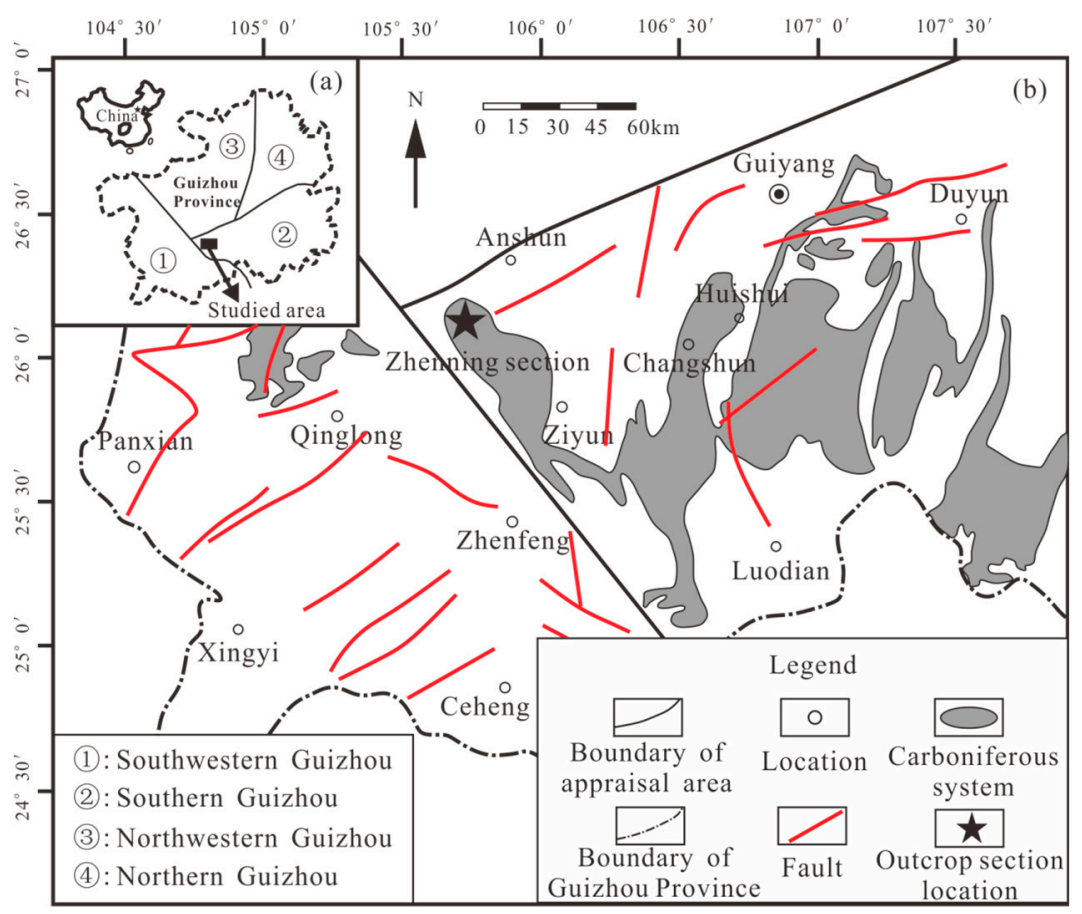

Figure 1. The simplified geologic map of the study area showing the location of sampling outcrop (star), southwest of Anshun city in Southern Guizhou, Southwest China. Base map modified from [27].

The Lower Carboniferous formations, unconformably overlying the Devonian strata, are composed of a basal carbonate succession (Yanguan Formation) and an overlying composite unit of siliciclastic rock and carbonate (Datang Formation) [22,23]. Above the regional unconformity, the Yanguan Formation (up to $440 \mathrm{~m}$ thick) is dominated by thick-layered dark-gray limestones and dolostones, locally intercalated with thin black shales/mudstones. The overlying Datang Formation consists of a thicker organic-rich lower part (maximum thickness up to $800 \mathrm{~m}$ ) characterized by black shales/mudstones intercalated with dark-gray marlstones/limestones and a thicker organic-poor upper part (maximum thickness up to $500 \mathrm{~m}$ ) dominated by dark-grey limestones and dolostones intercalated with black shales/marlstones [22,23]. This paper focuses on the lower part of the Datang Formation.

The investigated Zhenning section (N 25 $53^{\prime} 25.31^{\prime \prime}$, E $\left.105^{\circ} 51^{\prime} 23.98^{\prime \prime}\right)$ (Figure $1 \mathrm{~b}$ ) in this work is located about $30 \mathrm{~km}$ southwest of Anshun city and lies paleogeographically in an inter-platform basin $[18,20]$. At Zhenning County, the Datang Formation comprises in ascending order: (1) basal beds including $\sim 7 \mathrm{~m}$ of marlstone, $<2 \mathrm{~m}$ of mudstone, and a 6.5 -m-thick shale; (2) $\sim 4 \mathrm{~m}$ of marlstone (Figure 2a), a 4.5 -m-thick shale, $\sim 7 \mathrm{~m}$ of mudstone (Figure $2 \mathrm{~b}$ ); (3) $<1 \mathrm{~m}$ of marlstone, a 8 -m-thick shale, $\sim 4 \mathrm{~m}$ of limestone (not included in this study), and a 6-m-thick mudstone (Figure 2c); (4) $\sim 4 \mathrm{~m}$ of marlstone, $\sim 15 \mathrm{~m}$ of shale (Figure $2 \mathrm{~d}$ ), $\sim 3 \mathrm{~m}$ of limestone, and a 6.5 -m-thick mudstone.

The vertical lithological changes of the studied section show a well-developed stratigraphic cyclicity (Figure 3), which is generally considered to result from short-term orbitally-driven sea level variations $[28,29]$. The depositional cycle $(C)$ is the basic stratigraphic unit and consists of one component (one kind of lithology of the black rock series). Two or three depositional cycles vertically merge into one cycle set (CS) and then two such cycle sets further stack into one composite cycle set (CCS) (Figure 3). The cycle set is constituted of two parts: the lower marlstone/limestone and the upper shale/mudstone, apparently reflecting a marine transgression process. From the base to the top, four composite cycle sets are discerned in the investigated interval, being consistent with the TOC variations (Figure 3). 

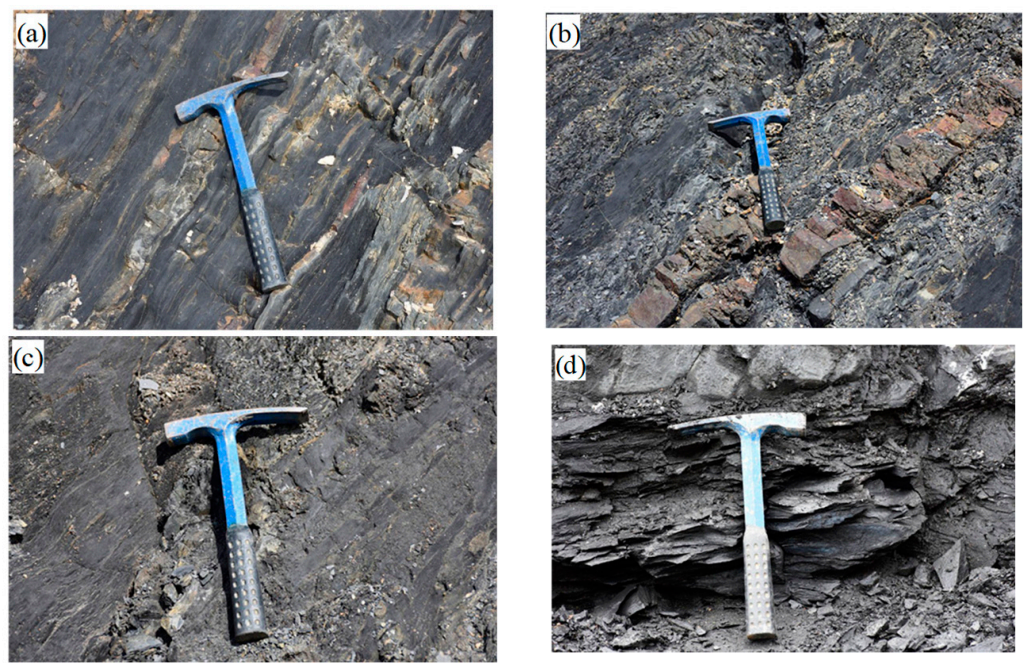

Figure 2. Outcrop view of sedimentary rocks from Zhenning section. (a) Dark-gray marlstone intercalated with thin limestone lamina; (b) Black mudstone intercalated with brown thin limestone laminae; (c) Black thick-layered mudstone with laminated bedding; (d) Black thick-layered shale with cross-layer fractures.

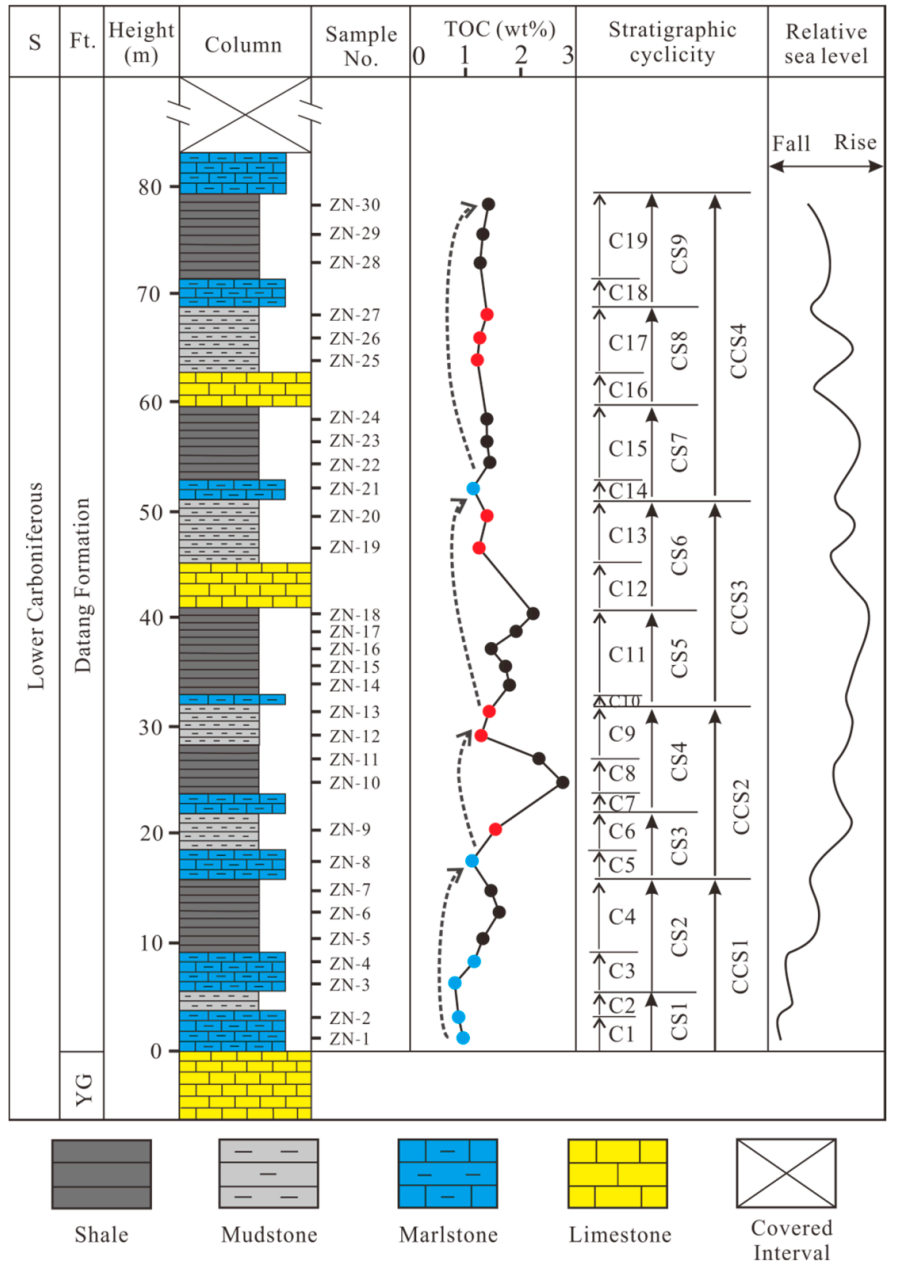

Figure 3. Stratigraphic column of the investigated section. Note the stratigraphic cyclicity and their superimposed patterns and inferred relative sea level fluctuations. Height represents the distance above the base of the Datang Formation. C, CS and CCS represent depositional cycle, cycle set and composite cycle set, respectively. YG stands for Yanguan Formation. 


\section{Samples and Methods}

\subsection{Samples}

Experimental samples for the analysis of TOC contents and elemental geochemistry in the Lower Carboniferous Datang Formation were collected systematically from a newly-cut roadside outcrop southwest of Anshun city named as Zhenning section (Figure 1b) in this study, and no published reports about it so far. Using sampling of vertical intervals of 2-3 $\mathrm{m}$, based on variations in lithology, a total of 30 rock samples were collected. The samples included: sixteen shales (ZN5-ZN7, ZN10-ZN11, ZN14-ZN18, ZN22-ZN24 and ZN28-ZN30), eight mudstones (ZN9, ZN12-ZN13, ZN19-ZN20 and ZN25-ZN27) and six marlstones (ZN1-ZN4, ZN8 and ZN21). Samples were saved in sample sacks to prevent external contamination. Table 1 and Figure 3 display more detailed information of the studied samples, including Formation, sample number, height and lithology.

\subsection{Methods}

Prior to analysis, all the studied samples were firstly washed and dried, and then were crushed and ground to a grain size smaller than 200 mesh $(75 \mu \mathrm{m})$ in an agate mortar for TOC, mineralogical compositions, major elements, TEs, and REEs analyses. All these analyses were performed on whole-rock powdered samples and conducted at the geochemistry laboratory of the Keyuan Engineering Testing Center of Sichuan Province, China.

An aliquot of $200 \mathrm{mg}$ of powdered samples for TOC contents were first treated with $10 \%$ (volume) hydrochloric acid $(\mathrm{HCl})$ at $60^{\circ} \mathrm{C}$ to dissolve carbonate, and whereafter rinsed with distillated water to wash the $\mathrm{HCl}$. Afterwards, the de-carbonated samples were dried for several hours at $50{ }^{\circ} \mathrm{C}$ and then analyzed using a LECO CS-400 analyzer. The analytical precision was better than $0.1 \%$.

The mineral compositions were determined by a Bruker D8 Discover X-ray diffractometer (XRD) with Ni-filtered $\mathrm{Cu}-\mathrm{K} \alpha$ radiation and a scintillation counter. Each XRD pattern was recorded over a $2 \theta$ interval of $5^{\circ}-70^{\circ}$, with a step size of $0.02^{\circ}$. Computer analysis of diffract grams could provide identification of minerals and semi-quantitative results of the weight percentage of each mineral type.

For major elements analysis, powdered samples were heated to $105^{\circ} \mathrm{C}$ to remove the adsorbed water before analysis and then heated to $920^{\circ} \mathrm{C}$ to analyze the LOI (loss on ignition). Then, $700 \mathrm{mg}$ of ashed samples and $7 \mathrm{~g}$ of $\mathrm{Li}_{2} \mathrm{~B}_{4} \mathrm{O}_{7}$ and $\mathrm{BLiO}_{2}$ mixture were fused at $1150{ }^{\circ} \mathrm{C}$ into a glass disc using an Analymate V8C instrument. Major elements were measured on the glass disc using a wavelength dispersive $\mathrm{X}$-ray fluorescence (XRF) spectrometer, following the procedures depicted by Goto and Tatsumi (1994) [30]. The reference material GSR5 was used simultaneously for monitoring the analytical accuracy and precision. The analytical precision for major elements was generally better than $5 \%$, and the detection limits were commonly lower than $30 \mathrm{mg} / \mathrm{kg}$.

For TEs and REEs analysis, the PE NexION 350X Inductively Coupled Plasma-Mass Spectrometry (ICP-MS) was used. An aliquot of $75 \mathrm{mg}$ of powdered samples were digested in a pressure-tight Teflon bomb. Each sample was weighed and dissolved using $3 \mathrm{~mL}$ of mixed acids ( $\mathrm{HF}: \mathrm{HCl}: \mathrm{HNO}_{3}=1: 1: 3$ ). The resulting liquid was permitted to react for $12-24 \mathrm{~h}$ at a high temperature (180-190 $\left.{ }^{\circ} \mathrm{C}\right)$. Further, after cooling, $1 \mathrm{~mL} \mathrm{HClO}_{4}$ was added and was reheated till dry to remove HF. Finally, the solution was then added $1 \mathrm{~mL}$ diluted $\mathrm{HNO}_{3}$ again and further diluted with double distilled water to a final volume of $100 \mathrm{~mL}$ with addition of $1 \mathrm{~mL} \mathrm{Rh}(500 \mathrm{ng} / \mathrm{mL})$ solution as an internal standard. Two reference materials GSR3 and GSR5 were applied simultaneously to supervising the analytical accuracy and precision. The analytical precision was better than $8 \%$ for TEs and REEs. 
Table 1. TOC, major element concentrations and their geochemical indicators of the target samples from Zhenning section (unit: $\mathrm{wt} \%$ ).

\begin{tabular}{|c|c|c|c|c|c|c|c|c|c|c|c|c|c|c|}
\hline Sample No. & Lithology & Height (m) & TOC & $\mathrm{SiO}_{2}$ & $\mathrm{Al}_{2} \mathrm{O}_{3}$ & $\mathrm{Fe}_{2} \mathrm{O}_{3}$ & $\mathrm{MgO}$ & $\mathrm{CaO}$ & $\mathrm{Na}_{2} \mathrm{O}$ & $\mathrm{K}_{2} \mathrm{O}$ & $\mathrm{MnO}$ & $\mathrm{TiO}_{2}$ & $\mathrm{P}_{2} \mathrm{O}_{5}$ & LOI \\
\hline ZN-1 & Marlstone & 1.2 & 0.97 & 40.37 & 12.24 & 6.7 & 1.83 & 24.48 & 1.55 & 3.36 & 0.047 & 0.83 & 0.051 & 8.07 \\
\hline $\mathrm{ZN}-2$ & Marlstone & 3.0 & 0.91 & 41.03 & 11.41 & 4.62 & 1.96 & 27.11 & 1.26 & 3.39 & 0.063 & 0.838 & 0.054 & 7.69 \\
\hline $\mathrm{ZN}-3$ & Marlstone & 6.0 & 0.90 & 41.73 & 10.87 & 4.61 & 1.97 & 26.33 & 1.07 & 3.39 & 0.066 & 0.843 & 0.054 & 8.99 \\
\hline $\mathrm{ZN}-4$ & Marlstone & 8.5 & 1.06 & 40.23 & 11.59 & 6.52 & 2.77 & 27.85 & 1.17 & 3.07 & 0.067 & 0.313 & 0.057 & 7.25 \\
\hline ZN-5 & Shale & 10.2 & 1.36 & 64.3 & 16.37 & 3.12 & 1.2 & 4.53 & 0.78 & 2.18 & 0.015 & 0.881 & 0.03 & 4.45 \\
\hline ZN-6 & Shale & 12.9 & 1.69 & 65.17 & 15.04 & 2.9 & 0.9 & 5.14 & 0.95 & 1.98 & 0.005 & 0.792 & 0.028 & 4.91 \\
\hline ZN-7 & Shale & 14.8 & 1.51 & 60.23 & 16.02 & 3.94 & 1.32 & 6.1 & 0.74 & 2.48 & 0.009 & 0.88 & 0.024 & 3.02 \\
\hline ZN-8 & Marlstone & 17.2 & 1.05 & 42.22 & 13.39 & 6.79 & 2.24 & 23.29 & 0.85 & 2.54 & 0.008 & 0.844 & 0.084 & 7.53 \\
\hline ZN-9 & Mudstone & 20.0 & 1.64 & 63.28 & 15.42 & 3.17 & 1.21 & 5.11 & 0.83 & 1.8 & 0.01 & 0.682 & 0.029 & 2.85 \\
\hline $\mathrm{ZN}-10$ & Shale & 24.5 & 2.83 & 63.11 & 16.4 & 3.59 & 1.03 & 5.09 & 1.23 & 1.35 & 0.007 & 0.584 & 0.021 & 3.78 \\
\hline ZN-11 & Shale & 27.0 & 2.29 & 60.76 & 15.69 & 4.76 & 1.78 & 4.61 & 0.84 & 2.33 & 0.012 & 0.825 & 0.041 & 3.82 \\
\hline $\mathrm{ZN}-12$ & Mudstone & 29.5 & 1.27 & 60.53 & 14.8 & 3.86 & 1.56 & 4.09 & 0.73 & 2.62 & 0.01 & 0.909 & 0.029 & 3.81 \\
\hline ZN-13 & Mudstone & 31.5 & 1.42 & 60.87 & 14.39 & 3.48 & 1.34 & 5.1 & 0.97 & 2.52 & 0.012 & 0.934 & 0.026 & 3.21 \\
\hline $\mathrm{ZN}-14$ & Shale & 33.5 & 1.82 & 55.49 & 15.06 & 4.38 & 1.9 & 5.09 & 1.24 & 3.13 & 0.008 & 1.087 & 0.04 & 4.82 \\
\hline $\mathrm{ZN}-15$ & Shale & 35.5 & 1.72 & 56.7 & 13.91 & 4.82 & 1.98 & 4.69 & 1.38 & 2.84 & 0.007 & 1.001 & 0.046 & 5.17 \\
\hline $\mathrm{ZN}-16$ & Shale & 37.0 & 1.47 & 59.2 & 13.07 & 4.41 & 2.07 & 4.37 & 0.95 & 2.71 & 0.007 & 0.881 & 0.033 & 3.43 \\
\hline ZN-17 & Shale & 38.7 & 1.93 & 56.24 & 13.84 & 4.64 & 1.55 & 3.95 & 0.85 & 2.66 & 0.003 & 1.044 & 0.03 & 5.33 \\
\hline ZN-18 & Shale & 40.2 & 2.12 & 56.77 & 14.18 & 4.13 & 2.18 & 4.09 & 1.06 & 2.58 & 0.012 & 1.045 & 0.043 & 4.57 \\
\hline ZN-19 & Mudstone & 46.5 & 1.19 & 64.52 & 15.27 & 4.22 & 1.74 & 4.81 & 1.13 & 2.66 & 0.003 & 0.976 & 0.028 & 4.71 \\
\hline $\mathrm{ZN}-20$ & Mudstone & 49.8 & 1.27 & 60.51 & 16.19 & 3.39 & 1.47 & 5.39 & 1.07 & 2.75 & 0.001 & 0.934 & 0.036 & 3.52 \\
\hline $\mathrm{ZN}-21$ & Marlstone & 52.0 & 1.08 & 41.25 & 13.21 & 4.95 & 2.2 & 27.15 & 0.6 & 2.82 & 0.002 & 0.902 & 0.035 & 7.69 \\
\hline $\mathrm{ZN}-22$ & Shale & 54.5 & 1.46 & 59.3 & 15.72 & 3.81 & 3.81 & 4.05 & 1.34 & 1.83 & 0.02 & 1.173 & 0.046 & 5.30 \\
\hline $\mathrm{ZN}-23$ & Shale & 56.0 & 1.42 & 61.83 & 15.01 & 3.54 & 3.78 & 4.48 & 0.94 & 1.74 & 0.021 & 1.073 & 0.044 & 4.95 \\
\hline $\mathrm{ZN}-24$ & Shale & 58.0 & 1.43 & 55.6 & 16.19 & 5.88 & 2.38 & 5.36 & 0.81 & 2.53 & 0.029 & 1.032 & 0.048 & 5.02 \\
\hline $\mathrm{ZN}-25$ & Mudstone & 63.2 & 1.14 & 52.25 & 18.56 & 5.7 & 2.55 & 4.61 & 1.12 & 2.3 & 0.043 & 0.803 & 0.043 & 5.21 \\
\hline $\mathrm{ZN}-26$ & Mudstone & 65.5 & 1.18 & 57.92 & 13.5 & 4.48 & 1.25 & 6.5 & 1.26 & 1.08 & 0.015 & 0.576 & 0.053 & 5.99 \\
\hline $\mathrm{ZN}-27$ & Mudstone & 68.0 & 1.38 & 65.45 & 13.83 & 2.25 & 1.4 & 5.34 & 1.21 & 1.82 & 0.003 & 0.406 & 0.015 & 5.79 \\
\hline $\mathrm{ZN}-28$ & Shale & 72.8 & 1.24 & 57.58 & 13.02 & 4.49 & 2.33 & 6.45 & 1.01 & 1.17 & 0.071 & 0.407 & 0.036 & 5.69 \\
\hline ZN-29 & Shale & 75.6 & 1.29 & 56.92 & 14.38 & 6.22 & 4.14 & 6.25 & 1.08 & 1.2 & 0.097 & 0.449 & 0.025 & 5.99 \\
\hline ZN-30 & Shale & 78.4 & 1.43 & 64.77 & 13.84 & 3.26 & 1.57 & 5.39 & 1.17 & 1.92 & 0.012 & 0.453 & 0.036 & 3.26 \\
\hline Average & & & 1.45 & 56.20 & 14.41 & 4.42 & 1.98 & 9.23 & 1.04 & 2.36 & 0.02 & 0.81 & 0.04 & 5.19 \\
\hline
\end{tabular}


Table 1. Cont.

\begin{tabular}{|c|c|c|c|c|}
\hline Sample No. & CIA & CIW & C-Value & $\mathrm{Al} / \mathrm{Na}$ \\
\hline $\mathrm{ZN}-1$ & / & / & 0.21 & 4.80 \\
\hline $\mathrm{ZN}-2$ & / & / & 0.14 & 5.51 \\
\hline ZN-3 & / & / & 0.14 & 6.18 \\
\hline $\mathrm{ZN}-4$ & / & / & 0.21 & 6.02 \\
\hline ZN-5 & 76.87 & 86.45 & 0.35 & 12.76 \\
\hline ZN-6 & 74.06 & 82.80 & 0.31 & 9.63 \\
\hline $\mathrm{ZN}-7$ & 75.79 & 86.81 & 0.36 & 13.16 \\
\hline $\mathrm{ZN}-8$ & / & / & 0.23 & 9.58 \\
\hline ZN-9 & 76.72 & 84.96 & 0.35 & 11.30 \\
\hline $\mathrm{ZN}-10$ & 74.86 & 80.21 & 0.40 & 8.11 \\
\hline ZN-11 & 74.80 & 85.03 & 0.49 & 11.36 \\
\hline $\mathrm{ZN}-12$ & 73.86 & 86.04 & 0.42 & 12.33 \\
\hline $\mathrm{ZN}-13$ & 70.86 & 81.85 & 0.34 & 9.02 \\
\hline $\mathrm{ZN}-14$ & 66.86 & 78.69 & 0.37 & 7.39 \\
\hline $\mathrm{ZN}-15$ & 64.63 & 75.40 & 0.43 & 6.13 \\
\hline $\mathrm{ZN}-16$ & 68.33 & 80.71 & 0.43 & 8.37 \\
\hline ZN-17 & 70.92 & 83.19 & 0.50 & 9.90 \\
\hline ZN-18 & 69.31 & 80.27 & 0.41 & 8.13 \\
\hline ZN-19 & 75.63 & 88.21 & 0.40 & 8.22 \\
\hline $\mathrm{ZN}-20$ & 76.77 & 89.38 & 0.31 & 9.20 \\
\hline $\mathrm{ZN}-21$ & / & / & 0.15 & 13.39 \\
\hline $\mathrm{ZN}-22$ & 71.11 & 78.10 & 0.35 & 7.13 \\
\hline $\mathrm{ZN}-23$ & 75.11 & 82.92 & 0.33 & 9.71 \\
\hline $\mathrm{ZN}-24$ & 74.98 & 85.87 & 0.52 & 12.15 \\
\hline $\mathrm{ZN}-25$ & 75.04 & 83.44 & 0.53 & 10.08 \\
\hline $\mathrm{ZN}-26$ & 71.76 & 76.51 & 0.44 & 6.52 \\
\hline $\mathrm{ZN}-27$ & 69.92 & 77.65 & 0.23 & 6.95 \\
\hline $\mathrm{ZN}-28$ & 73.94 & 79.67 & 0.41 & 7.84 \\
\hline ZN-29 & 74.78 & 80.19 & 0.50 & 8.10 \\
\hline ZN-30 & 76.37 & 86.26 & 0.32 & 7.19 \\
\hline Average & 71.20 & 81.50 & 0.35 & 8.87 \\
\hline
\end{tabular}

Note: CIA: Chemical Index of Alteration; CIW: Chemical Index of Weathering; C-Value: the ratio of $\Sigma(\mathrm{Fe}+\mathrm{Mn}+\mathrm{Cr}+\mathrm{Ni}+\mathrm{V}+\mathrm{Co}) / \Sigma(\mathrm{Ca}+\mathrm{Mg}+\mathrm{Sr}+\mathrm{Ba}+\mathrm{K}+\mathrm{Na})$. 


\section{Results}

\subsection{TOC}

TOC contents for all the 30 samples are presented in Table 1 and Figure 3. TOC contents vary from $0.90 \%$ to $2.83 \%$, with an average of $1.45 \%$. Additionally, there are systematic variations in TOC which accord with stratigraphic cyclicity, with lower values (mean 1.0\%) in the basal marlstone and higher values (mean 1.56\%) in the upper shale or mudstone of each cycle set (Figure 3).

\subsection{Major Elements}

The concentrations of major element oxides in the black rock series samples from Zhenning section are presented in Table 1, including average values and LOI (loss on ignition). The major chemical compositions are $\mathrm{SiO}_{2}(56.20 \%), \mathrm{Al}_{2} \mathrm{O}_{3}(14.41 \%), \mathrm{CaO}(9.23 \%), \mathrm{Fe}_{2} \mathrm{O}_{3}(4.42 \%), \mathrm{MgO}(1.98 \%), \mathrm{K}_{2} \mathrm{O}$ $(2.36 \%)$, and $\mathrm{Na}_{2} \mathrm{O}(1.04 \%)$. The absolute abundances of other major element oxides are obviously less than $1 \%$ and include $\mathrm{TiO}_{2}(0.81 \%), \mathrm{P}_{2} \mathrm{O}_{5}(0.04 \%)$, and $\mathrm{MnO}(0.02 \%)$. Compared to marlstones, shales and mudstones are enriched in $\mathrm{SiO}_{2}(59.96 \%)$ and $\mathrm{Al}_{2} \mathrm{O}_{3}(15.05 \%)$, which are strongly linked with the amount of terrestrial detrital inputs [31]. Contrary to shales and mudstones, marlstones are enriched in the absolute abundances of $\mathrm{CaO}(26.04 \%), \mathrm{Fe}_{2} \mathrm{O}_{3}(5.70 \%)$ and $\mathrm{K}_{2} \mathrm{O}(3.10 \%)$. The whole major element oxide concentrations of the target samples vary slightly and show no distinct vertical variation rules, reflecting that the heterogeneity of black rock series samples is relatively weak (Figure 4). Particularly, subtle fluctuations of $\mathrm{Al}_{2} \mathrm{O}_{3}$ and $\mathrm{TiO}_{2}$ concentrations imply that terrestrial detrital input is relatively stable [3,32] (Figure 4).
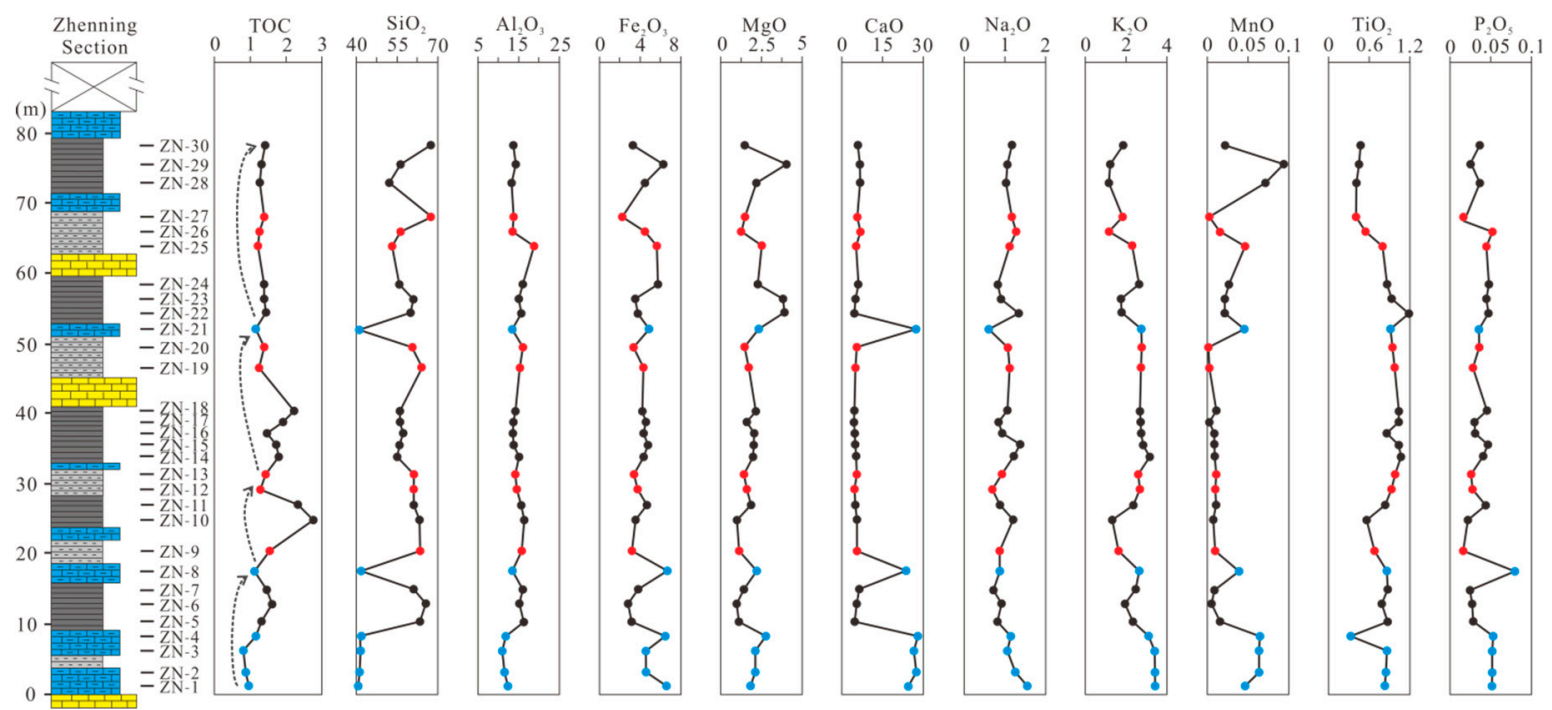

Figure 4. Vertical variation of major elements of the black rock series samples (unit: $w \mathrm{t} \%$ ).

\subsection{Trace Elements}

The selected TEs compositions of the black rock series samples are shown in terms of their average concentrations and standard deviation values in Table 2. On average, elemental content data show that the most abundant TEs in this black rock series are Sr (255.2 ppm), Ba (182.0 ppm), V (171.8 ppm), $\mathrm{Cr}(165.0 \mathrm{ppm}), \mathrm{Rb}(104.9 \mathrm{ppm}), \mathrm{Zr}(116.9 \mathrm{ppm})$ and Ni (57.2 ppm). The concentrations of other TEs are lower than 50 ppm (Table 2). 
Table 2. Concentrations of selected trace elements (ppm) and their ratios of Datang Formation black rock series.

\begin{tabular}{|c|c|c|c|c|c|c|c|c|}
\hline Sample No. & Sc & V & $\mathrm{Cr}$ & Co & $\mathbf{N i}$ & $\mathrm{Cu}$ & $\mathrm{Ga}$ & $\mathbf{R b}$ \\
\hline $\mathrm{ZN}-1$ & $19.2 \pm 1.5$ & $193.3 \pm 9.7$ & $163.5 \pm 6.5$ & $46.3 \pm 2.3$ & $74.0 \pm 3.7$ & $17.7 \pm 0.9$ & $29.7 \pm 1.2$ & $162.3 \pm 4.9$ \\
\hline $\mathrm{ZN}-2$ & $16.7 \pm 1.3$ & $176.0 \pm 8.8$ & $146.7 \pm 5.9$ & $6.5 \pm 0.3$ & $54.9 \pm 2.7$ & $18.8 \pm 0.9$ & $26.6 \pm 1.1$ & $141.4 \pm 4.2$ \\
\hline ZN-3 & $17.2 \pm 1.4$ & $194.6 \pm 9.7$ & $152.3 \pm 6.1$ & $5.2 \pm 0.3$ & $53.0 \pm 2.6$ & $21.4 \pm 1.1$ & $27.4 \pm 1.1$ & $152.8 \pm 4.6$ \\
\hline $\mathrm{ZN}-4$ & $10.3 \pm 0.8$ & $88.9 \pm 4.4$ & $75.0 \pm 3.0$ & $11.4 \pm 0.6$ & $60.5 \pm 3.0$ & $14.6 \pm 0.7$ & $9.1 \pm 0.4$ & $52.3 \pm 1.6$ \\
\hline ZN-5 & $11.5 \pm 0.9$ & $190.4 \pm 9.5$ & $173.9 \pm 7.0$ & $7.1 \pm 0.4$ & $60.4 \pm 3.0$ & $27.0 \pm 1.4$ & $20.3 \pm 0.8$ & $102.2 \pm 3.1$ \\
\hline ZN-6 & $8.7 \pm 0.7$ & $150.67 \pm 7.5$ & $145.8 \pm 5.8$ & $3.8 \pm 0.2$ & $63.2 \pm 3.2$ & $15.6 \pm 0.8$ & $17.5 \pm 0.7$ & $78.6 \pm 2.4$ \\
\hline $\mathrm{ZN-7}$ & $12.1 \pm 1.0$ & $167.9 \pm 8.4$ & $160.4 \pm 6.4$ & $8.3 \pm 0.4$ & $58.7 \pm 2.9$ & $33.3 \pm 1.7$ & $22.2 \pm 0.9$ & $105.7 \pm 3.2$ \\
\hline ZN-8 & $20.5 \pm 1.6$ & $191.7 \pm 9.6$ & $173.8 \pm 7.0$ & $25.9 \pm 1.43$ & $51.5 \pm 2.6$ & $12.2 \pm 0.6$ & $18.8 \pm 0.8$ & $113.7 \pm 3.4$ \\
\hline ZN-9 & $12.0 \pm 1.0$ & $125.6 \pm 6.3$ & $128.2 \pm 5.1$ & $11.7 \pm 0.6$ & $57.7 \pm 2.9$ & $26.8 \pm 1.3$ & $15.9 \pm 0.6$ & $85.7 \pm 2.6$ \\
\hline $\mathrm{ZN}-10$ & $10.5 \pm 0.3$ & $111.7 \pm 5.6$ & $110.2 \pm 4.4$ & $10.4 \pm 0.5$ & $66.5 \pm 3.3$ & $32.4 \pm 1.6$ & $13.2 \pm 0.5$ & $63.2 \pm 1.9$ \\
\hline $\mathrm{ZN}-11$ & $14.4 \pm 1.1$ & $158.5 \pm 7.9$ & $151.7 \pm 6.1$ & $10.6 \pm 0.5$ & $75.2 \pm 3.8$ & $27.8 \pm 1.4$ & $20.6 \pm 0.8$ & $102.3 \pm 3.1$ \\
\hline $\mathrm{ZN}-12$ & $14.5 \pm 1.2$ & $155.6 \pm 7.8$ & $154.9 \pm 6.2$ & $11.0 \pm 0.5$ & $61.0 \pm 3.1$ & $22.0 \pm 1.1$ & $22.1 \pm 0.9$ & $113.3 \pm 3.4$ \\
\hline $\mathrm{ZN}-13$ & $15.0 \pm 1.2$ & $199.5 \pm 10.0$ & $192.0 \pm 7.7$ & $14.2 \pm 0.7$ & $89.3 \pm 4.5$ & $29.7 \pm 1.5$ & $22.4 \pm 0.9$ & $119.7 \pm 3.6$ \\
\hline $\mathrm{ZN}-14$ & $18.9 \pm 1.5$ & $244.1 \pm 12.2$ & $203.0 \pm 8.1$ & $13.9 \pm 0.7$ & $82.6 \pm 4.1$ & $27.8 \pm 1.4$ & $27.2 \pm 1.1$ & $151.2 \pm 4.5$ \\
\hline $\mathrm{ZN}-15$ & $17.5 \pm 1.4$ & $215.6 \pm 10.8$ & $191.4 \pm 7.7$ & $14.6 \pm 0.7$ & $103.6 \pm 5.2$ & $30.2 \pm 1.5$ & $24.4 \pm 1.0$ & $136.2 \pm 4.1$ \\
\hline $\mathrm{ZN}-16$ & $15.5 \pm 1.2$ & $181.6 \pm 9.1$ & $165.5 \pm 6.6$ & $12.5 \pm 0.6$ & $74.0 \pm 3.7$ & $36.7 \pm 1.8$ & $23.3 \pm 0.9$ & $118.2 \pm 3.5$ \\
\hline ZN-17 & $16.6 \pm 1.3$ & $211.4 \pm 10.6$ & $184.7 \pm 7.4$ & $16.9 \pm 0.8$ & $72.7 \pm 3.6$ & $29.0 \pm 1.4$ & $23.0 \pm 0.9$ & $119.4 \pm 3.6$ \\
\hline $\mathrm{ZN}-18$ & $18.6 \pm 1.5$ & $229.8 \pm 11.5$ & $209.8 \pm 8.4$ & $9.9 \pm 0.5$ & $67.4 \pm 3.3$ & $20.1 \pm 1.0$ & $23.7 \pm 0.9$ & $127.2 \pm 3.8$ \\
\hline ZN-19 & $19.5 \pm 1.6$ & $202.7 \pm 10.1$ & $186.1 \pm 7.4$ & $16.5 \pm 0.8$ & $27.0 \pm 1.4$ & $14.2 \pm 0.7$ & $20.2 \pm 0.8$ & $120.5 \pm 3.6$ \\
\hline $\mathrm{ZN}-20$ & $16.0 \pm 1.3$ & $223.9 \pm 11.2$ & $204.8 \pm 8.2$ & $16.2 \pm 0.8$ & $38.2 \pm 1.9$ & $10.9 \pm 0.5$ & $22.0 \pm 0.9$ & $135.3 \pm 4.1$ \\
\hline $\mathrm{ZN}-21$ & $19.5 \pm 1.6$ & $235.3 \pm 11.8$ & $198.8 \pm 8.0$ & $16.7 \pm 0.8$ & $35.6 \pm 1.8$ & $10.5 \pm 0.5$ & $22.6 \pm 0.9$ & $137.2 \pm 4.1$ \\
\hline ZN-22 & $15.4 \pm 1.2$ & $200.0 \pm 10.0$ & $196.3 \pm 7.9$ & $16.1 \pm 0.8$ & $58.8 \pm 2.9$ & $11.5 \pm 0.6$ & $22.4 \pm 0.9$ & $91.5 \pm 2.7$ \\
\hline $\mathrm{ZN}-23$ & $14.1 \pm 1.1$ & $169.3 \pm 8.5$ & $148.6 \pm 5.9$ & $11.3 \pm 0.6$ & $51.1 \pm 2.6$ & $18.6 \pm 0.9$ & $20.8 \pm 0.8$ & $74.0 \pm 2.2$ \\
\hline $\mathrm{ZN}-24$ & $16.9 \pm 1.3$ & $203.6 \pm 10.2$ & $173.8 \pm 7.0$ & $8.7 \pm 0.4$ & $39.6 \pm 2.0$ & $13.7 \pm 0.7$ & $21.6 \pm 0.9$ & $129.2 \pm 3.9$ \\
\hline $\mathrm{ZN}-25$ & $18.1 \pm 1.4$ & $171.2 \pm 8.6$ & $142.3 \pm 6.7$ & $6.5 \pm 0.3$ & $34.6 \pm 1.7$ & $14.5 \pm 0.7$ & $21.4 \pm 0.9$ & $102.0 \pm 3.1$ \\
\hline $\mathrm{ZN}-26$ & $10.9 \pm 0.9$ & $131.2 \pm 6.6$ & $216.4 \pm 8.7$ & $8.3 \pm 0.4$ & $39.2 \pm 2.0$ & $12.2 \pm 0.6$ & $12.4 \pm 0.5$ & $51.7 \pm 1.6$ \\
\hline $\mathrm{ZN}-27$ & $6.1 \pm 0.9$ & $103.6 \pm 5.2$ & $278.4 \pm 11.1$ & $5.0 \pm 0.3$ & $26.1 \pm 1.3$ & $7.2 \pm 0.4$ & $8.2 \pm 0.3$ & $45.5 \pm 1.4$ \\
\hline $\mathrm{ZN}-28$ & $9.4 \pm 0.7$ & $100.1 \pm 5.0$ & $96.1 \pm 3.8$ & $9.5 \pm 0.5$ & $50.4 \pm 2.5$ & $14.5 \pm 0.7$ & $9.2 \pm 0.4$ & $56.6 \pm 1.7$ \\
\hline ZN-29 & $11.3 \pm 0.9$ & $118.2 \pm 5.9$ & $111.5 \pm 4.5$ & $11.0 \pm 0.6$ & $52.6 \pm 2.6$ & $14.7 \pm 0.7$ & $9.6 \pm 0.4$ & $67.0 \pm 2.0$ \\
\hline $\mathrm{ZN}-30$ & $9.1 \pm 0.7$ & $107.1 \pm 5.4$ & $115.0 \pm 4.6$ & $5.5 \pm 0.3$ & $36.2 \pm 1.8$ & $12.0 \pm 0.6$ & $13.0 \pm 0.5$ & $92.1 \pm 2.8$ \\
\hline Average & 14.5 & 171.8 & 165 & 12.4 & 57.2 & 19.9 & 19.7 & 104.9 \\
\hline STDEV & 3.78 & 43.28 & 40.7 & 7.79 & 17.96 & 8.02 & 5.75 & 32.21 \\
\hline
\end{tabular}


Table 2. Cont.

\begin{tabular}{|c|c|c|c|c|c|c|c|}
\hline Sample No. & $\mathrm{Sr}$ & $\mathrm{Zr}$ & Mo & Cs & Ва & Th & $\mathbf{U}$ \\
\hline $\mathrm{ZN}-1$ & $243.4 \pm 7.3$ & $117.3 \pm 4.7$ & $1.6 \pm 0.1$ & $14.2 \pm 0.7$ & $89.7 \pm 3.6$ & $21.9 \pm 0.9$ & $3.2 \pm 0.1$ \\
\hline $\mathrm{ZN}-2$ & $206.3 \pm 6.2$ & $112.0 \pm 4.5$ & $1.1 \pm 0.1$ & $12.7 \pm 0.6$ & $176.4 \pm 7.1$ & $20.5 \pm 0.8$ & $3.3 \pm 0.1$ \\
\hline ZN-3 & $222.4 \pm 6.7$ & $115.7 \pm 4.6$ & $1.0 \pm 0$ & $12.5 \pm 0.6$ & $168.7 \pm 6.7$ & $19.3 \pm 0.8$ & $3.2 \pm 0.1$ \\
\hline $\mathrm{ZN}-4$ & $464.1 \pm 13.9$ & $41.0 \pm 1.6$ & $2.4 \pm 0.1$ & $4.3 \pm 0.2$ & $80.9 \pm 3.2$ & $8.2 \pm 0.3$ & $3.0 \pm 0.1$ \\
\hline ZN-5 & $177.7 \pm 5.3$ & $117.7 \pm 4.7$ & $1.7 \pm 0.1$ & $7.0 \pm 0.4$ & $177.4 \pm 7.1$ & $16.0 \pm 0.6$ & $3.4 \pm 0.1$ \\
\hline ZN-6 & $155.2 \pm 4.7$ & $106.4 \pm 4.3$ & $1.9 \pm 0.1$ & $6.5 \pm 0.3$ & $169.7 \pm 6.8$ & $16.3 \pm 0.7$ & $3.3 \pm 0.1$ \\
\hline ZN-7 & $164.9 \pm 4.9$ & $123.0 \pm 4.9$ & $1.8 \pm 0.1$ & $8.0 \pm 0.4$ & $198.2 \pm 7.9$ & $17.7 \pm 0.7$ & $4.3 \pm 0.2$ \\
\hline $\mathrm{ZN}-8$ & $181.1 \pm 5.4$ & $130.1 \pm 5.2$ & $1.6 \pm 0.1$ & $8.0 \pm 0.4$ & $180.8 \pm 7.2$ & $19.9 \pm 0.8$ & $3.1 \pm 0.1$ \\
\hline ZN-9 & $126.5 \pm 3.8$ & $100.2 \pm 4.0$ & $2.4 \pm 0.1$ & $5.1 \pm 0.3$ & $103.9 \pm 4.2$ & $13.4 \pm 0.5$ & $3.1 \pm 0.1$ \\
\hline ZN-10 & $109.4 \pm 3.3$ & $96.1 \pm 3.8$ & $3.9 \pm 0.2$ & $4.5 \pm 0.2$ & $322.7 \pm 12.9$ & $10.6 \pm 0.4$ & $4.1 \pm 0.2$ \\
\hline $\mathrm{ZN}-11$ & $154.23 \pm 4.6$ & $116.2 \pm 4.6$ & $3.2 \pm 0.2$ & $8.0 \pm 0.4$ & $191.5 \pm 7.7$ & $17.6 \pm 0.7$ & $4.4 \pm 0.2$ \\
\hline $\mathrm{ZN}-12$ & $171.1 \pm 5.1$ & $125.2 \pm 5.0$ & $2.0 \pm 0.1$ & $8.5 \pm 0.4$ & $187.2 \pm 7.5$ & $18.8 \pm 0.8$ & $4.3 \pm 0.2$ \\
\hline $\mathrm{ZN}-13$ & $206.5 \pm 6.2$ & $143.4 \pm 5.7$ & $2.5 \pm 0.1$ & $9.3 \pm 0.5$ & $206.6 \pm 8.3$ & $18.9 \pm 0.8$ & $4.1 \pm 0.2$ \\
\hline $\mathrm{ZN}-14$ & $213.2 \pm 6.4$ & $151.1 \pm 6.0$ & $1.8 \pm 0.1$ & $10.5 \pm 0.5$ & $240.7 \pm 9.6$ & $20.6 \pm 0.8$ & $4.9 \pm 0.2$ \\
\hline ZN-15 & $202.6 \pm 6.1$ & $153.8 \pm 6.2$ & $1.9 \pm 0.1$ & $11.1 \pm 0.6$ & $242.2 \pm 9.7$ & $19.2 \pm 0.8$ & $4.5 \pm 0.2$ \\
\hline ZN-16 & $188.1 \pm 5.6$ & $125.7 \pm 5.0$ & $1.7 \pm 0.1$ & $9.3 \pm 0.5$ & $212.3 \pm 8.5$ & $17.8 \pm 0.7$ & $4.1 \pm 0.2$ \\
\hline $\mathrm{ZN}-17$ & $196.3 \pm 5.9$ & $149.8 \pm 6.0$ & $2.6 \pm 0.1$ & $9.9 \pm 0.5$ & $216.6 \pm 8.7$ & $21.4 \pm 0.9$ & $5.2 \pm 0.2$ \\
\hline $\mathrm{ZN}-18$ & $219.5 \pm 6.6$ & $156.4 \pm 6.3$ & $2.3 \pm 0.1$ & $9.2 \pm 0.5$ & $198.5 \pm 7.9$ & $19.1 \pm 0.8$ & $4.5 \pm 0.2$ \\
\hline ZN-19 & $156.0 \pm 4.7$ & $143.1 \pm 5.7$ & $1.2 \pm 0.1$ & $7.9 \pm 0.4$ & $195.9 \pm 7.8$ & $20.9 \pm 0.8$ & $3.7 \pm 0.1$ \\
\hline $\mathrm{ZN}-20$ & $176.4 \pm 5.3$ & $133.4 \pm 5.3$ & $1.2 \pm 0.1$ & $8.2 \pm 0.4$ & $195.0 \pm 7.8$ & $21.9 \pm 0.9$ & $3.9 \pm 0.2$ \\
\hline $\mathrm{ZN}-21$ & $169.2 \pm 5.1$ & $134.2 \pm 5.4$ & $1.8 \pm 0.1$ & $8.0 \pm 0.4$ & $188.6 \pm 7.5$ & $21.5 \pm 0.9$ & $3.6 \pm 0.1$ \\
\hline ZN-22 & $199.6 \pm 6.0$ & $167.6 \pm 6.7$ & $1.7 \pm 0.1$ & $6.9 \pm 0.3$ & $227.3 \pm 9.1$ & $25.2 \pm 1.0$ & $4.4 \pm 0.2$ \\
\hline $\mathrm{ZN}-23$ & $158.8 \pm 4.8$ & $131.8 \pm 5.3$ & $1.4 \pm 0.1$ & $6.2 \pm 0.3$ & $109.4 \pm 4.4$ & $24.1 \pm 1.0$ & $4.0 \pm 0.2$ \\
\hline $\mathrm{ZN}-24$ & $316.4 \pm 9.5$ & $139.5 \pm 5.6$ & $1.6 \pm 0.1$ & $9.5 \pm 0.5$ & $193.7 \pm 7.7$ & $22.2 \pm 0.9$ & $4.0 \pm 0.2$ \\
\hline $\mathrm{ZN}-25$ & $296.8 \pm 8.9$ & $110.2 \pm 4.4$ & $2.7 \pm 0.1$ & $7.8 \pm 0.4$ & $189.1 \pm 7.6$ & $17.9 \pm 0.7$ & $3.2 \pm 0.1$ \\
\hline $\mathrm{ZN}-26$ & $573.8 \pm 17.2$ & $90.5 \pm 3.6$ & $2.3 \pm 0.1$ & $8.6 \pm 0.5$ & $171.6 \pm 6.9$ & $13.4 \pm 0.5$ & $2.6 \pm 0.1$ \\
\hline $\mathrm{ZN}-27$ & $356.7 \pm 10.7$ & $68.0 \pm 2.7$ & $3.1 \pm 0.2$ & $5.9 \pm 0.3$ & $151.4 \pm 6.1$ & $8.1 \pm 0.3$ & $5.8 \pm 0.2$ \\
\hline ZN-28 & $704.9 \pm 21.1$ & $65.2 \pm 2.6$ & $1.7 \pm 0.1$ & $5.3 \pm 0.3$ & $154.7 \pm 6.2$ & $8.8 \pm 0.4$ & $3.1 \pm 0.1$ \\
\hline ZN-29 & $795.4 \pm 23.9$ & $75.3 \pm 3.0$ & $1.4 \pm 0.1$ & $5.6 \pm 0.3$ & $157.3 \pm 6.3$ & $10.6 \pm 0.4$ & $3.1 \pm 0.1$ \\
\hline ZN-30 & $149.7 \pm 4.5$ & $68.8 \pm 2.8$ & $3.4 \pm 0.2$ & $4.6 \pm 0.2$ & $161.6 \pm 6.5$ & $7.7 \pm 0.3$ & $3.5 \pm 0.1$ \\
\hline Average & 255.2 & 116.9 & 2 & 8.1 & 182 & 17.3 & 3.8 \\
\hline STDEV & 163.95 & 30.13 & 0.7 & 2.44 & 46.97 & 4.91 & 0.72 \\
\hline
\end{tabular}


Table 2. Cont

\begin{tabular}{|c|c|c|c|c|c|c|c|c|c|c|c|c|}
\hline Sample No. & U/Th & $\mathrm{V} / \mathrm{Cr}$ & $\mathrm{Ni} / \mathrm{Co}$ & $\mathrm{V} /(\mathrm{V}+\mathrm{Ni})$ & $\mathrm{La} / \mathrm{Sc}$ & La/Co & $\mathrm{Th} / \mathrm{Sc}$ & $\mathrm{Co} / \mathrm{Th}$ & $\mathrm{Cr} / \mathrm{Th}$ & $\mathrm{La} / \mathrm{Th}$ & $\mathrm{Sr} / \mathrm{Cu}$ & $\mathrm{Ga} / \mathrm{Rb}$ \\
\hline $\mathrm{ZN}-1$ & 0.15 & 1.18 & 1.6 & 0.72 & 2.63 & 1.09 & 1.14 & 2.11 & 7.45 & 2.3 & 13.74 & 0.18 \\
\hline $\mathrm{ZN}-2$ & 0.16 & 1.2 & 8.49 & 0.76 & 2.84 & 7.35 & 1.22 & 0.32 & 7.17 & 2.32 & 10.97 & 0.19 \\
\hline $\mathrm{ZN}-3$ & 0.17 & 1.28 & 10.11 & 0.79 & 2.66 & 8.75 & 1.12 & 0.27 & 7.88 & 2.37 & 10.39 & 0.18 \\
\hline $\mathrm{ZN}-4$ & 0.37 & 1.18 & 5.33 & 0.6 & 3.12 & 2.85 & 0.79 & 1.38 & 9.14 & 3.93 & 31.91 & 0.17 \\
\hline ZN-5 & 0.21 & 1.1 & 8.54 & 0.76 & 3.15 & 5.11 & 1.39 & 0.44 & 10.89 & 2.26 & 6.58 & 0.2 \\
\hline $\mathrm{ZN}-6$ & 0.2 & 1.03 & 16.45 & 0.7 & 4.38 & 9.9 & 1.88 & 0.24 & 8.93 & 2.33 & 9.96 & 0.22 \\
\hline $\mathrm{ZN}-7$ & 0.24 & 1.05 & 7.04 & 0.74 & 3.41 & 4.94 & 1.46 & 0.47 & 9.07 & 2.33 & 4.95 & 0.21 \\
\hline ZN-8 & 0.15 & 1.1 & 1.99 & 0.79 & 1.76 & 1.39 & 0.97 & 1.3 & 8.74 & 1.81 & 14.8 & 0.17 \\
\hline ZN-9 & 0.23 & 0.98 & 4.93 & 0.69 & 2.69 & 2.76 & 1.11 & 0.88 & 9.59 & 2.42 & 4.72 & 0.19 \\
\hline $\mathrm{ZN}-10$ & 0.38 & 1.01 & 6.4 & 0.63 & 2.59 & 2.62 & 1.01 & 0.98 & 10.4 & 2.56 & 3.38 & 0.21 \\
\hline ZN-11 & 0.25 & 1.04 & 7.09 & 0.68 & 3.13 & 4.24 & 1.23 & 0.6 & 8.62 & 2.56 & 5.55 & 0.2 \\
\hline $\mathrm{ZN}-12$ & 0.23 & 1 & 5.56 & 0.72 & 2.82 & 3.72 & 1.3 & 0.58 & 8.25 & 2.17 & 7.77 & 0.2 \\
\hline ZN-13 & 0.22 & 1.04 & 6.3 & 0.69 & 2.94 & 3.12 & 1.26 & 0.75 & 10.16 & 2.34 & 6.96 & 0.19 \\
\hline $\mathrm{ZN}-14$ & 0.24 & 1.2 & 5.96 & 0.75 & 2.87 & 3.9 & 1.09 & 0.67 & 9.88 & 2.63 & 7.68 & 0.18 \\
\hline ZN-15 & 0.24 & 1.13 & 7.08 & 0.68 & 3.06 & 3.67 & 1.1 & 0.76 & 9.96 & 2.79 & 6.7 & 0.18 \\
\hline $\mathrm{ZN}-16$ & 0.23 & 1.1 & 5.9 & 0.71 & 2.96 & 3.66 & 1.15 & 0.71 & 9.31 & 2.58 & 5.13 & 0.2 \\
\hline ZN-17 & 0.24 & 1.14 & 4.31 & 0.74 & 2.73 & 2.69 & 1.29 & 0.79 & 8.64 & 2.12 & 6.77 & 0.19 \\
\hline ZN-18 & 0.23 & 1.1 & 6.83 & 0.77 & 2.48 & 4.65 & 1.03 & 0.52 & 10.98 & 2.4 & 10.91 & 0.19 \\
\hline ZN-19 & 0.18 & 1.09 & 1.63 & 0.88 & 1.82 & 2.14 & 1.07 & 0.79 & 8.9 & 1.69 & 11.01 & 0.17 \\
\hline $\mathrm{ZN}-20$ & 0.18 & 1.09 & 2.36 & 0.85 & 2.43 & 2.41 & 1.37 & 0.74 & 9.34 & 1.78 & 16.16 & 0.16 \\
\hline $\mathrm{ZN}-21$ & 0.17 & 1.18 & 2.14 & 0.87 & 1.96 & 2.3 & 1.1 & 0.78 & 9.25 & 1.78 & 16.18 & 0.16 \\
\hline $\mathrm{ZN}-22$ & 0.18 & 1.02 & 3.65 & 0.77 & 2.87 & 2.74 & 1.64 & 0.64 & 7.78 & 1.75 & 17.31 & 0.24 \\
\hline $\mathrm{ZN}-23$ & 0.17 & 1.14 & 4.51 & 0.77 & 2.87 & 3.58 & 1.71 & 0.47 & 6.16 & 1.68 & 8.55 & 0.28 \\
\hline $\mathrm{ZN}-24$ & 0.18 & 1.17 & 4.55 & 0.84 & 2.51 & 4.88 & 1.31 & 0.39 & 7.83 & 1.91 & 23.13 & 0.17 \\
\hline $\mathrm{ZN}-25$ & 0.18 & 1.2 & 5.31 & 0.83 & 1.95 & 5.42 & 0.99 & 0.36 & 7.95 & 1.97 & 20.49 & 0.21 \\
\hline $\mathrm{ZN}-26$ & 0.2 & 0.61 & 4.74 & 0.77 & 3.28 & 4.32 & 1.22 & 0.62 & 16.21 & 2.68 & 47.23 & 0.24 \\
\hline $\mathrm{ZN}-27$ & 0.71 & 0.37 & 5.22 & 0.8 & 3.65 & 4.43 & 1.34 & 0.62 & 34.31 & 2.73 & 49.75 & 0.18 \\
\hline $\mathrm{ZN}-28$ & 0.35 & 1.04 & 5.28 & 0.67 & 2.59 & 2.56 & 0.93 & 1.08 & 10.91 & 2.78 & 48.71 & 0.16 \\
\hline ZN-29 & 0.29 & 1.06 & 4.76 & 0.69 & 2.51 & 2.56 & 0.94 & 1.05 & 10.57 & 2.68 & 54.17 & 0.14 \\
\hline $\mathrm{ZN}-30$ & 0.45 & 0.93 & 6.56 & 0.75 & 2.59 & 4.3 & 0.85 & 0.71 & 14.86 & 3.06 & 12.51 & 0.14 \\
\hline Average & 0.25 & 1.06 & 5.69 & 0.75 & 2.78 & 3.94 & 0.85 & 0.73 & 10.3 & 2.36 & 16.47 & 0.19 \\
\hline
\end{tabular}

Note: STDEV means standard deviation. 
In order to further characterize the enrichment degree of TE in sediments, Enrichment Factor (EF) has been universally applied [3,33]. $\mathrm{EF}_{\text {element }}$ is determined by normalizing each element to aluminium (Al), which is supposed to stand for the detrital influx, and then comparing these ratios to their equivalent of a normal shale, such as average shale [3,33,34], namely:

$$
\mathrm{EF}_{\text {element }}=(\text { element } / \mathrm{Al})_{\text {sample }} /(\text { element } / \mathrm{Al})_{\text {average shale }}
$$

Among the above equation, the ratio in the numerator is for the investigated samples, while that in the denominator is for an average shale (data from [35]). Generally, when $\mathrm{EF}_{\text {element }}>1$, the TE is enriched relative to average shale, whereas $\mathrm{EF}_{\text {element }}<1$ indicates depletion [36].

Based on the EFs averages (Table 3), Cr (2.12), Cs (1.71), Th (1.67), V (1.53), Sc (1.29) and Ga (1.20) are slightly enriched, which may be associated with the accumulation of organic matter and/or clay minerals [37,38]. In contrast, $\mathrm{U}(0.91), \mathrm{Mo}(0.90), \mathrm{Rb}(0.87), \mathrm{Zr}(0.85), \mathrm{Co}(0.75), \mathrm{Cu}(0.51)$ and $\mathrm{Ba}(0.36)$ are relatively depleted, being likely owing to the weathering of black rock series [38,39]. With regard to rock type, the marlstone samples show the highest average EFs values for most of the selected TEs (Figure 5). The shale and mudstone samples have comparative EFs values, but show distinctly lower than those of the marlstone samples (Figure 5).

Table 3. Enrichment Factors (EFs) for the selected trace elements from the studied section.

\begin{tabular}{|c|c|c|c|c|c|c|c|c|c|c|c|c|c|c|c|}
\hline Sample No. & Al (\%) & $\mathrm{EF}_{\mathrm{Sc}}$ & $\mathrm{EF}_{\mathrm{V}}$ & $\mathrm{EF}_{\mathrm{Cr}}$ & $\mathrm{EF}_{\mathrm{Co}}$ & $\mathrm{EF}_{\mathrm{Ni}}$ & $\mathrm{EF}_{\mathrm{Cu}}$ & $\mathrm{EF}_{\mathrm{Ga}}$ & $\mathrm{EF}_{\mathrm{Rb}}$ & $\mathrm{EF}_{\mathrm{Sr}}$ & $\mathrm{EF}_{\mathrm{Mo}}$ & $\mathrm{EF}_{\mathrm{Cs}}$ & $\mathrm{EF}_{\mathrm{Ba}}$ & $\mathrm{EF}_{\mathrm{Th}}$ & $\mathrm{EF}_{\mathrm{U}}$ \\
\hline ZN-1 & 6.48 & 2.02 & 2.03 & 2.48 & 3.32 & 1.49 & 0.54 & 2.13 & 1.58 & 1.11 & 0.85 & 3.53 & 0.21 & 2.49 & 0.10 \\
\hline ZN-2 & 6.04 & 1.88 & 1.98 & 2.39 & 0.50 & 1.18 & 0.61 & 2.05 & 1.48 & 1.01 & 0.60 & 3.39 & 0.44 & 2.49 & 0.11 \\
\hline ZN-3 & 5.75 & 2.04 & 2.30 & 2.60 & 0.42 & 1.20 & 0.73 & 2.21 & 1.68 & 1.14 & 0.57 & 3.51 & 0.45 & 2.47 & 0.11 \\
\hline ZN-4 & 6.14 & 1.15 & 0.99 & 1.20 & 0.86 & 1.28 & 0.47 & 0.69 & 0.54 & 2.23 & 1.34 & 1.14 & 0.20 & 0.98 & 0.10 \\
\hline ZN-5 & 8.67 & 0.90 & 1.49 & 1.97 & 0.38 & 0.91 & 0.61 & 1.09 & 0.74 & 0.60 & 0.68 & 1.31 & 0.31 & 1.35 & 0.08 \\
\hline ZN-6 & 7.96 & 0.74 & 1.29 & 1.80 & 0.22 & 1.03 & 0.38 & 1.02 & 0.62 & 0.57 & 0.83 & 1.32 & 0.32 & 1.51 & 0.08 \\
\hline ZN-7 & 8.48 & 0.97 & 1.35 & 1.86 & 0.46 & 0.90 & 0.77 & 1.22 & 0.79 & 0.57 & 0.72 & 1.52 & 0.36 & 1.53 & 0.10 \\
\hline ZN-8 & 7.09 & 1.97 & 1.84 & 2.41 & 1.70 & 0.94 & 0.34 & 1.23 & 1.01 & 0.75 & 0.76 & 1.82 & 0.39 & 2.06 & 0.09 \\
\hline ZN-9 & 8.16 & 1.00 & 1.05 & 1.54 & 0.67 & 0.92 & 0.65 & 0.90 & 0.66 & 0.46 & 1.02 & 1.01 & 0.19 & 1.20 & 0.07 \\
\hline ZN-10 & 8.68 & 0.82 & 0.87 & 1.25 & 0.56 & 1.00 & 0.73 & 0.70 & 0.46 & 0.37 & 1.55 & 0.83 & 0.57 & 0.90 & 0.09 \\
\hline ZN-11 & 8.31 & 1.18 & 1.30 & 1.79 & 0.59 & 1.18 & 0.66 & 1.15 & 0.78 & 0.55 & 1.32 & 1.55 & 0.35 & 1.56 & 0.10 \\
\hline ZN-12 & 7.84 & 1.26 & 1.35 & 1.94 & 0.65 & 1.01 & 0.55 & 1.31 & 0.91 & 0.64 & 0.88 & 1.75 & 0.36 & 1.76 & 0.11 \\
\hline ZN-13 & 7.62 & 1.34 & 1.78 & 2.48 & 0.87 & 1.52 & 0.76 & 1.37 & 0.99 & 0.80 & 1.13 & 1.97 & 0.41 & 1.82 & 0.11 \\
\hline ZN-14 & 7.97 & 1.61 & 2.08 & 2.50 & 0.81 & 1.35 & 0.68 & 1.59 & 1.20 & 0.79 & 0.79 & 2.13 & 0.46 & 1.90 & 0.12 \\
\hline ZN-15 & 7.36 & 1.62 & 1.99 & 2.55 & 0.92 & 1.83 & 0.81 & 1.54 & 1.17 & 0.81 & 0.89 & 2.44 & 0.50 & 1.92 & 0.12 \\
\hline ZN-16 & 6.92 & 1.52 & 1.78 & 2.35 & 0.84 & 1.39 & 1.04 & 1.57 & 1.08 & 0.80 & 0.87 & 2.17 & 0.47 & 1.89 & 0.12 \\
\hline ZN-17 & 7.33 & 1.54 & 1.96 & 2.48 & 1.07 & 1.29 & 0.78 & 1.46 & 1.03 & 0.79 & 1.20 & 2.17 & 0.45 & 2.15 & 0.14 \\
\hline ZN-18 & 7.51 & 1.68 & 2.08 & 2.75 & 0.61 & 1.17 & 0.53 & 1.47 & 1.07 & 0.86 & 1.07 & 1.98 & 0.40 & 1.87 & 0.12 \\
\hline ZN-19 & 8.08 & 1.64 & 1.70 & 2.26 & 0.95 & 0.43 & 0.34 & 1.16 & 0.94 & 0.57 & 0.52 & 1.57 & 0.37 & 1.90 & 0.09 \\
\hline $\mathrm{ZN}-20$ & 8.57 & 1.27 & 1.78 & 2.35 & 0.88 & 0.58 & 0.25 & 1.20 & 1.00 & 0.61 & 0.49 & 1.54 & 0.35 & 1.88 & 0.09 \\
\hline ZN-21 & 6.99 & 1.89 & 2.29 & 2.79 & 1.11 & 0.66 & 0.29 & 1.50 & 1.24 & 0.71 & 0.90 & 1.84 & 0.41 & 2.26 & 0.10 \\
\hline $\mathrm{ZN}-22$ & 8.32 & 1.26 & 1.63 & 2.32 & 0.90 & 0.92 & 0.27 & 1.25 & 0.69 & 0.71 & 0.72 & 1.33 & 0.42 & 2.23 & 0.10 \\
\hline ZN-23 & 7.95 & 1.21 & 1.45 & 1.84 & 0.66 & 0.84 & 0.46 & 1.22 & 0.59 & 0.59 & 0.60 & 1.26 & 0.21 & 2.23 & 0.10 \\
\hline $\mathrm{ZN}-24$ & 8.57 & 1.34 & 1.61 & 1.99 & 0.47 & 0.60 & 0.31 & 1.17 & 0.95 & 1.09 & 0.62 & 1.78 & 0.34 & 1.90 & 0.09 \\
\hline ZN-25 & 9.83 & 1.25 & 1.18 & 1.42 & 0.31 & 0.46 & 0.29 & 1.01 & 0.66 & 0.89 & 0.94 & 1.28 & 0.29 & 1.34 & 0.06 \\
\hline ZN-26 & 7.15 & 1.04 & 1.25 & 2.97 & 0.54 & 0.71 & 0.33 & 0.81 & 0.46 & 2.37 & 1.10 & 1.95 & 0.37 & 1.37 & 0.07 \\
\hline ZN-27 & 7.32 & 0.56 & 0.96 & 3.74 & 0.32 & 0.46 & 0.19 & 0.52 & 0.39 & 1.44 & 1.47 & 1.30 & 0.32 & 0.81 & 0.15 \\
\hline ZN-28 & 6.89 & 0.93 & 0.99 & 1.37 & 0.64 & 0.95 & 0.41 & 0.62 & 0.52 & 3.01 & 0.84 & 1.23 & 0.34 & 0.94 & 0.09 \\
\hline ZN-29 & 7.61 & 1.01 & 1.06 & 1.44 & 0.67 & 0.90 & 0.38 & 0.59 & 0.56 & 3.08 & 0.64 & 1.19 & 0.32 & 1.02 & 0.08 \\
\hline ZN-30 & 7.33 & 0.85 & 0.99 & 1.54 & 0.35 & 0.64 & 0.32 & 0.83 & 0.79 & 0.60 & 1.58 & 1.01 & 0.34 & 0.78 & 0.09 \\
\hline Average & 7.63 & 1.32 & 1.55 & 2.15 & 0.78 & 0.99 & 0.52 & 1.22 & 0.89 & 1.09 & 0.92 & 1.76 & 0.36 & 1.68 & 0.10 \\
\hline
\end{tabular}

\subsection{Rare Earth Elements}

The REE concentrations and associated geochemical indicators for the analyzed samples are listed in Table 4 . The total REE ( $\sum$ REE) concentrations of the 30 black rock series varied from 120.74 to $206.49 \mathrm{ppm}$, with an average of $174.85 \mathrm{ppm}$ (Table 4), being close to that of the North American Shale Composite (NASC) (173.21 ppm; [40]). The average value of REE concentrations for the black rock series is slightly lower than that of the Post-Archean Average Australian Shale (PAAS) (183.03 
ppm; [32]) and slightly higher than that of the Upper Continental Crust (UCC) (146.4 ppm; [32]). In this work, the NASC was adopted to normalize the REEs.

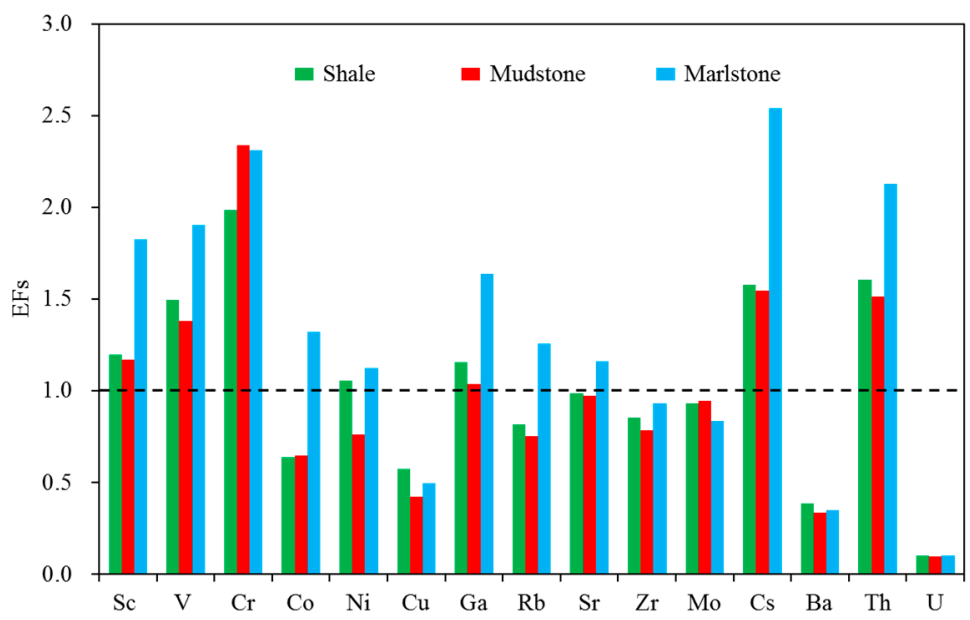

Figure 5. EFs diagram of the selected trace elements in the Datang Formation black rock series. A horizontal line $(\mathrm{EF}=1)$ highlighting an element enrichment or depletion.

Figure 6 displays the NASC-normalized REE distribution patterns for three different lithologies from Zhenning section. The REE distribution patterns of these three lithologies appear to have similar trends, being characterized by sloping light REE (LREE, from $\mathrm{La}$ to $\mathrm{Eu}$ ) and relatively flat heavy REE (HREE, from $\mathrm{Gd}$ to $\mathrm{Lu}$ ) with slightly negative Ce anomaly $\left((\mathrm{Ce} / \mathrm{Ce})_{\mathrm{N}}\right.$ ranging from 0.83 to 1.01 , with an average of 0.91$)$ and relatively obvious negative Eu anomaly $\left(\left(\mathrm{Eu} / \mathrm{Eu}^{*}\right)_{\mathrm{N}}\right.$ ranging from 0.60 to 1.10 , with an average of 0.84 ). The NASC-normalized distribution patterns of these three lithologies reveal that black rock series in Southern Guizhou are characterized by LREE enrichment and relative HREE depletion. In other words, the total contents of LREE ( $L$ LREE) are higher than those of HREE ( $\sum$ HREE), being in accordance with the $\sum$ LREE/ $\sum$ HREE ratio values higher than 1 (4.47-15.25, on average of 10.68; Table 4). The extremely uniform REEs distribution patterns imply that the REEs in black rock series have originated from the same terrestrial detrital input and are also likely to be controlled by similar depositional environments.

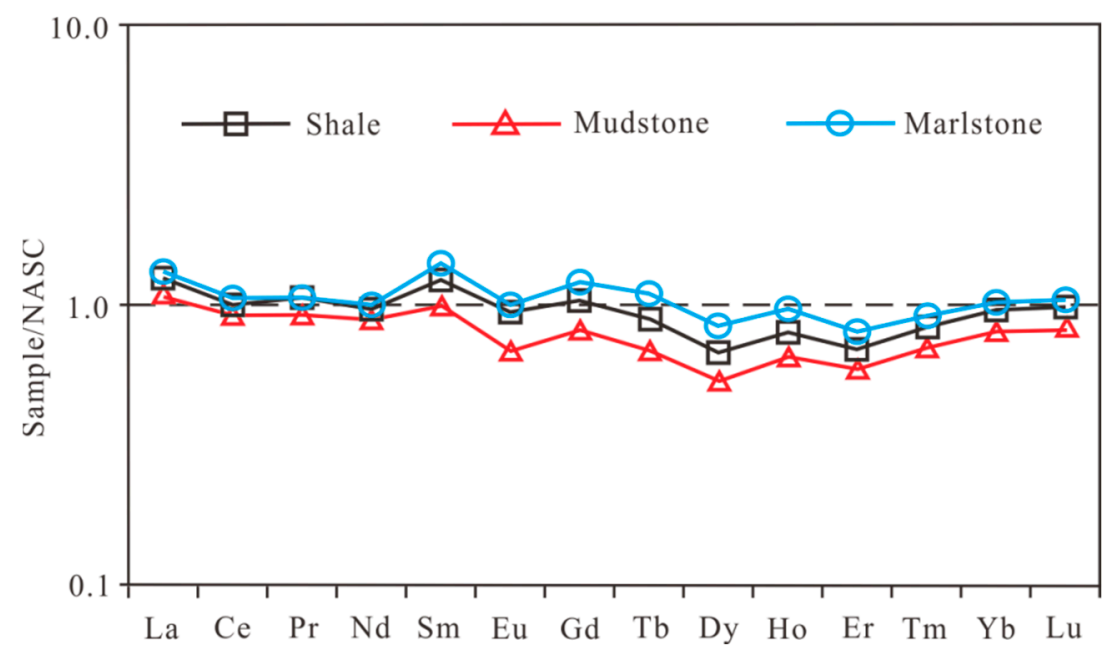

Figure 6. NASC-normalized REE distribution patterns for three different lithologies. 
Table 4. REE concentrations (ppm) and calculated REE ratios of the 30 black rock series samples from Zhenning section.

\begin{tabular}{|c|c|c|c|c|c|c|c|c|c|c|c|c|c|c|}
\hline Sample No. & La & $\mathrm{Ce}$ & Pr & $\mathrm{Nd}$ & $\mathrm{Sm}$ & Eu & Gd & $\mathbf{T b}$ & Dy & Ho & Er & Tm & $\mathrm{Yb}$ & Lu \\
\hline $\mathrm{ZN}-1$ & 50.56 & 89.87 & 9.6 & 35.43 & 5.87 & 0.95 & 4.48 & 0.57 & 3.02 & 0.67 & 2.06 & 0.37 & 2.64 & 0.42 \\
\hline $\mathrm{ZN}-2$ & 47.52 & 82.36 & 8.95 & 32.96 & 5.42 & 0.81 & 3.9 & 0.51 & 2.81 & 0.63 & 1.9 & 0.35 & 2.45 & 0.39 \\
\hline ZN-3 & 45.86 & 80.22 & 8.83 & 33.06 & 5.38 & 0.82 & 4.17 & 0.54 & 2.87 & 0.64 & 1.97 & 0.35 & 2.51 & 0.39 \\
\hline $\mathrm{ZN}-4$ & 32.29 & 52.68 & 6.44 & 27.48 & 8.85 & 1.6 & 9.2 & 1.54 & 7.86 & 1.52 & 3.77 & 0.59 & 3.85 & 0.6 \\
\hline ZN-5 & 36.14 & 62.72 & 7.29 & 28.02 & 5.01 & 0.78 & 3.69 & 0.52 & 2.69 & 0.59 & 1.75 & 0.31 & 2.25 & 0.36 \\
\hline ZN-6 & 38.01 & 67.49 & 7.6 & 28.52 & 4.84 & 0.69 & 3.27 & 0.4 & 2.07 & 0.44 & 1.44 & 0.25 & 1.85 & 0.29 \\
\hline ZN-7 & 41.15 & 68.29 & 8.09 & 30.5 & 4.83 & 0.7 & 3.5 & 0.42 & 2.36 & 0.56 & 1.78 & 0.32 & 2.36 & 0.37 \\
\hline ZN-8 & 35.96 & 59.7 & 8.9 & 37.59 & 10.44 & 2.17 & 9.53 & 1.6 & 8.07 & 1.49 & 3.72 & 0.61 & 4.1 & 0.62 \\
\hline ZN-9 & 32.3 & 55.76 & 7.27 & 30.56 & 6.95 & 1.49 & 7.21 & 1.07 & 5.42 & 1.1 & 2.98 & 0.51 & 3.54 & 0.56 \\
\hline $\mathrm{ZN}-10$ & 27.16 & 43.67 & 5.4 & 20.56 & 3.44 & 0.52 & 2.79 & 0.36 & 1.88 & 0.4 & 1.27 & 0.22 & 1.64 & 0.26 \\
\hline $\mathrm{ZN}-11$ & 44.98 & 74.94 & 9.29 & 36.13 & 6.32 & 1.07 & 4.74 & 0.65 & 3.43 & 0.73 & 2.22 & 0.39 & 2.82 & 0.46 \\
\hline $\mathrm{ZN}-12$ & 40.8 & 72.02 & 8.44 & 32.39 & 5.89 & 0.94 & 4.57 & 0.64 & 3.33 & 0.74 & 2.3 & 0.42 & 2.97 & 0.48 \\
\hline $\mathrm{ZN}-13$ & 44.15 & 78.32 & 9.17 & 34.49 & 5.98 & 0.84 & 3.91 & 0.45 & 2.11 & 0.46 & 1.55 & 0.29 & 2.21 & 0.36 \\
\hline ZN-14 & 54.09 & 97.13 & 11.86 & 48.97 & 11.52 & 2.74 & 12.04 & 1.84 & 9.43 & 1.94 & 5.15 & 0.87 & 5.92 & 0.95 \\
\hline ZN-15 & 53.65 & 98.1 & 12.07 & 50.66 & 13.1 & 3.23 & 13.79 & 2.16 & 10.95 & 2.22 & 5.79 & 0.95 & 6.38 & 1.03 \\
\hline $\mathrm{ZN}-16$ & 45.85 & 83.5 & 9.81 & 39.39 & 8.19 & 1.65 & 7.89 & 1.16 & 6.03 & 1.26 & 3.46 & 0.59 & 4.05 & 0.65 \\
\hline ZN-17 & 45.33 & 82.73 & 9.04 & 33.6 & 5.73 & 0.85 & 4.13 & 0.53 & 2.89 & 0.64 & 1.98 & 0.36 & 2.69 & 0.43 \\
\hline ZN-18 & 45.92 & 85.97 & 10.16 & 40.05 & 8.24 & 1.39 & 5.99 & 0.84 & 4.1 & 0.82 & 2.36 & 0.42 & 2.99 & 0.47 \\
\hline ZN-19 & 35.41 & 79.61 & 7.3 & 27.73 & 4.94 & 0.93 & 4.31 & 0.67 & 3.6 & 0.8 & 2.36 & 0.42 & 2.92 & 0.46 \\
\hline ZN-20 & 38.94 & 89.53 & 8.08 & 29.53 & 4.61 & 0.73 & 3.51 & 0.43 & 2.31 & 0.54 & 1.68 & 0.31 & 2.3 & 0.36 \\
\hline ZN-21 & 38.25 & 97.92 & 8.65 & 32.86 & 6.23 & 1.15 & 5.78 & 0.85 & 4.6 & 0.99 & 2.8 & 0.49 & 3.3 & 0.53 \\
\hline $\mathrm{ZN}-22$ & 44.2 & 90.65 & 8.3 & 29.77 & 4.61 & 0.75 & 3.71 & 0.45 & 2.4 & 0.54 & 1.81 & 0.34 & 2.48 & 0.41 \\
\hline $\mathrm{ZN}-23$ & 40.55 & 82.39 & 7.59 & 27.28 & 4.22 & 0.65 & 3.35 & 0.42 & 2.26 & 0.51 & 1.64 & 0.31 & 2.31 & 0.38 \\
\hline $\mathrm{ZN}-24$ & 42.43 & 89.06 & 8.36 & 30.91 & 5.27 & 0.77 & 4.04 & 0.51 & 2.65 & 0.59 & 1.87 & 0.34 & 2.51 & 0.4 \\
\hline ZN-25 & 35.3 & 72.38 & 7.1 & 26.8 & 4.52 & 0.76 & 3.62 & 0.47 & 2.61 & 0.56 & 1.75 & 0.31 & 2.26 & 0.36 \\
\hline ZN-26 & 35.75 & 64.35 & 7.5 & 29.91 & 5.36 & 0.8 & 4.68 & 0.67 & 3.66 & 0.79 & 2.26 & 0.37 & 2.51 & 0.38 \\
\hline $\mathrm{ZN}-27$ & 22.16 & 33.84 & 3.87 & 14.81 & 2.52 & 0.3 & 2.21 & 0.32 & 1.91 & 0.42 & 1.25 & 0.21 & 1.44 & 0.22 \\
\hline $\mathrm{ZN}-28$ & 24.45 & 38.21 & 6.09 & 27.12 & 5.94 & 1.25 & 5.08 & 0.76 & 3.37 & 0.69 & 1.81 & 0.34 & 2.37 & 0.39 \\
\hline $\mathrm{ZN}-29$ & 28.27 & 41.39 & 6.18 & 23.73 & 5.11 & 1.09 & 4.71 & 0.71 & 3.86 & 0.76 & 2 & 0.37 & 2.48 & 0.42 \\
\hline ZN-30 & 23.68 & 57.31 & 5.65 & 21.02 & 3.22 & 0.43 & 2.56 & 0.36 & 2.11 & 0.47 & 1.43 & 0.27 & 1.92 & 0.31 \\
\hline NASC $^{a}$ & 32 & 73 & 7.9 & 33 & 5.7 & 1.24 & 5.2 & 0.85 & 5.8 & 1.04 & 3.4 & 0.5 & 3.1 & 0.48 \\
\hline
\end{tabular}


Table 4. Cont.

\begin{tabular}{|c|c|c|c|c|c|c|c|c|c|c|}
\hline Sample No. & $\Sigma \mathrm{REE}$ & $\Sigma$ LREE & $\Sigma$ HREE & $\mathrm{L} / \mathrm{H}$ & $\left(\mathrm{La} / \mathrm{La}^{*}\right)_{\mathrm{N}}$ & $\left(\mathrm{Ce} / \mathrm{Ce}^{*}\right)_{N}$ & $\left(\operatorname{Pr} / \operatorname{Pr}^{*}\right)_{N}$ & $\left(E \mathbf{E} / \mathrm{Eu}^{*}\right)_{\mathrm{N}}$ & $(\mathrm{La} / \mathrm{Yb})_{\mathrm{N}}$ & $(\mathrm{Gd} / \mathrm{Yb})_{\mathrm{N}}$ \\
\hline $\mathrm{ZN}-1$ & 206.49 & 192.27 & 14.22 & 13.52 & 1.04 & 0.87 & 1.06 & 0.84 & 1.86 & 1.01 \\
\hline $\mathrm{ZN}-2$ & 190.93 & 178 & 12.92 & 13.78 & 1.06 & 0.87 & 1.06 & 0.78 & 1.87 & 0.95 \\
\hline $\mathrm{ZN}-3$ & 187.61 & 174.17 & 13.44 & 12.96 & 1.05 & 0.88 & 1.07 & 0.79 & 1.77 & 0.99 \\
\hline $\mathrm{ZN}-4$ & 158.26 & 129.34 & 28.93 & 4.47 & 1.31 & 0.89 & 1.05 & 0.79 & 0.81 & 1.43 \\
\hline ZN-5 & 152.12 & 139.96 & 12.16 & 11.51 & 1.07 & 0.9 & 1.08 & 0.8 & 1.55 & 0.97 \\
\hline ZN-6 & 157.17 & 147.15 & 10.02 & 14.69 & 1.03 & 0.89 & 1.08 & 0.77 & 1.98 & 1.05 \\
\hline ZN-7 & 165.24 & 153.56 & 11.68 & 13.15 & 1.06 & 0.88 & 1.1 & 0.76 & 1.7 & 0.88 \\
\hline $\mathrm{ZN}-8$ & 184.49 & 154.76 & 29.74 & 5.2 & 1.01 & 1 & 1.15 & 0.95 & 0.85 & 1.39 \\
\hline ZN-9 & 156.72 & 134.33 & 22.39 & 6 & 1.12 & 0.95 & 1.09 & 0.98 & 0.89 & 1.22 \\
\hline $\mathrm{ZN}-10$ & 109.57 & 100.76 & 8.81 & 11.44 & 1.06 & 0.89 & 1.11 & 0.78 & 1.6 & 1.02 \\
\hline ZN-11 & 188.18 & 172.74 & 15.44 & 11.19 & 1.04 & 0.91 & 1.11 & 0.87 & 1.55 & 1 \\
\hline $\mathrm{ZN}-12$ & 175.94 & 160.49 & 15.45 & 10.39 & 1.02 & 0.91 & 1.09 & 0.81 & 1.32 & 0.92 \\
\hline $\mathrm{ZN}-13$ & 184.29 & 172.95 & 11.34 & 15.25 & 1 & 0.91 & 1.09 & 0.78 & 1.94 & 1.06 \\
\hline $\mathrm{ZN}-14$ & 264.47 & 226.32 & 38.15 & 5.93 & 1.1 & 0.94 & 1.07 & 1.07 & 0.88 & 1.21 \\
\hline ZN-15 & 274.09 & 230.8 & 43.28 & 5.33 & 1.11 & 0.95 & 1.06 & 1.1 & 0.82 & 1.29 \\
\hline ZN-16 & 213.49 & 188.4 & 25.09 & 7.51 & 1.07 & 0.93 & 1.06 & 0.94 & 1.09 & 1.16 \\
\hline $\mathrm{ZN}-17$ & 190.92 & 177.27 & 13.65 & 12.99 & 1.03 & 0.89 & 1.06 & 0.77 & 1.63 & 0.91 \\
\hline ZN-18 & 209.74 & 191.74 & 18 & 10.65 & 0.99 & 0.95 & 1.08 & 0.86 & 1.48 & 1.19 \\
\hline ZN-19 & 171.45 & 155.91 & 15.55 & 10.03 & 1.03 & 0.91 & 0.95 & 0.89 & 1.18 & 0.88 \\
\hline $\mathrm{ZN}-20$ & 182.85 & 171.42 & 11.44 & 14.98 & 0.95 & 0.91 & 0.96 & 0.83 & 1.65 & 0.91 \\
\hline $\mathrm{ZN}-21$ & 204.4 & 185.07 & 19.34 & 9.57 & 0.92 & 0.96 & 0.94 & 0.88 & 1.12 & 1.04 \\
\hline $\mathrm{ZN}-22$ & 190.44 & 178.29 & 12.16 & 14.66 & 1.02 & 0.86 & 0.98 & 0.85 & 1.73 & 0.89 \\
\hline $\mathrm{ZN}-23$ & 173.85 & 162.69 & 11.16 & 14.58 & 1.04 & 0.86 & 0.98 & 0.79 & 1.72 & 0.86 \\
\hline $\mathrm{ZN}-24$ & 189.71 & 176.8 & 12.91 & 13.69 & 1.02 & 0.89 & 0.98 & 0.76 & 1.64 & 0.96 \\
\hline $\mathrm{ZN}-25$ & 158.79 & 146.87 & 11.93 & 12.31 & 1.02 & 0.9 & 1 & 0.86 & 1.51 & 0.96 \\
\hline $\mathrm{ZN}-26$ & 158.98 & 143.66 & 15.31 & 9.38 & 1.09 & 0.92 & 1.06 & 0.72 & 1.38 & 1.11 \\
\hline $\mathrm{ZN}-27$ & 85.5 & 77.51 & 7.99 & 9.7 & 1.21 & 0.83 & 1.08 & 0.6 & 1.47 & 0.91 \\
\hline $\mathrm{ZN}-28$ & 117.87 & 103.06 & 14.81 & 6.96 & 1.13 & 1.01 & 1.15 & 1.02 & 1 & 1.29 \\
\hline ZN-29 & 121.08 & 105.77 & 15.31 & 6.91 & 0.98 & 0.94 & 1.21 & 1 & 1.1 & 1.14 \\
\hline ZN-30 & 120.74 & 111.31 & 9.43 & 11.8 & 0.84 & 0.99 & 1.01 & 0.68 & 1.19 & 0.79 \\
\hline Average & 174.85 & 158.11 & 16.74 & 10.68 & 1.05 & 0.91 & 1.06 & 0.84 & 1.41 & 1.05 \\
\hline
\end{tabular}

Note: $\Sigma$ LREE: total concentrations of light rare earth elements (LREE $=\mathrm{La}+\mathrm{C}$

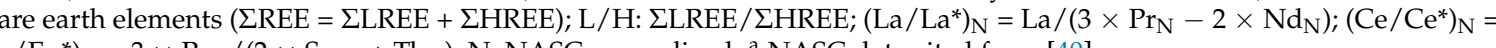

$\operatorname{Pr}_{\mathrm{N}} /\left(\mathrm{La}_{\mathrm{N}}+\operatorname{Pr}_{\mathrm{N}}\right) ;\left(\operatorname{Pr} / \mathrm{Pr}^{*}\right)_{\mathrm{N}}=2 \times \operatorname{Pr}_{\mathrm{N}} /\left(\mathrm{Ce}_{\mathrm{N}}+\mathrm{Nd}_{\mathrm{N}}\right) ;\left(\mathrm{Eu} / \mathrm{Eu}^{*}\right)_{\mathrm{N}}=3 \times \operatorname{Pr}_{\mathrm{N}} /\left(2 \times \mathrm{Sm}_{\mathrm{N}}+\mathrm{Tb}_{\mathrm{N}}\right) ; \mathrm{N}$ : NASC-normalized. ${ }^{\text {a }}$ NASC data cited from [40]. 
Except for two samples (ZN-9 and $\mathrm{ZN}-28,\left(\mathrm{Ce} / \mathrm{Ce}^{*}\right)_{\mathrm{N}}=1.00$ and 1.01, respectively), all the other samples from Datang Formation show slightly negative Ce anomaly (Table 4 and Figure 6). Compared among the three lithologies, they have comparative $\left(\mathrm{Ce} / \mathrm{Ce}^{*}\right)_{N}$ values, mirroring the weak heterogeneity during the black rock series deposition. Since the Ce anomaly in marine sediments is susceptible to the anomalous concentrations of La, interpretation of Ce anomaly is difficult and intricate [6,41]. The vast majority of highly negative Ce anomalies are resulted from highly positive La anomalies in marine sediments $[6,25,41]$, and most of the analyzed samples in this study exhibit slightly positive La anomalies $\left(\left(\mathrm{La} / \mathrm{La}^{*}\right)_{\mathrm{N}}=1.05\right)$. In order to determine the aberrant $(\mathrm{Ce} / \mathrm{Ce})_{\mathrm{N}}$ values resulting from a real Ce anomaly or representing an anomalous La concentration, the cross plot of $\left(\operatorname{Pr} / \mathrm{Pr}^{*}\right)_{\mathrm{N}}$ vs. $\left(\mathrm{Ce} / \mathrm{Ce}^{*}\right)_{\mathrm{N}}$ was widely used [6,41] (Figure 7). Except for three points, the rest of our data are clustered in the field IIIb (Figure 7), illustrating that the $\left(\mathrm{Ce} / \mathrm{Ce}^{*}\right)_{N}$ ratios mainly result from the real Ce anomaly.

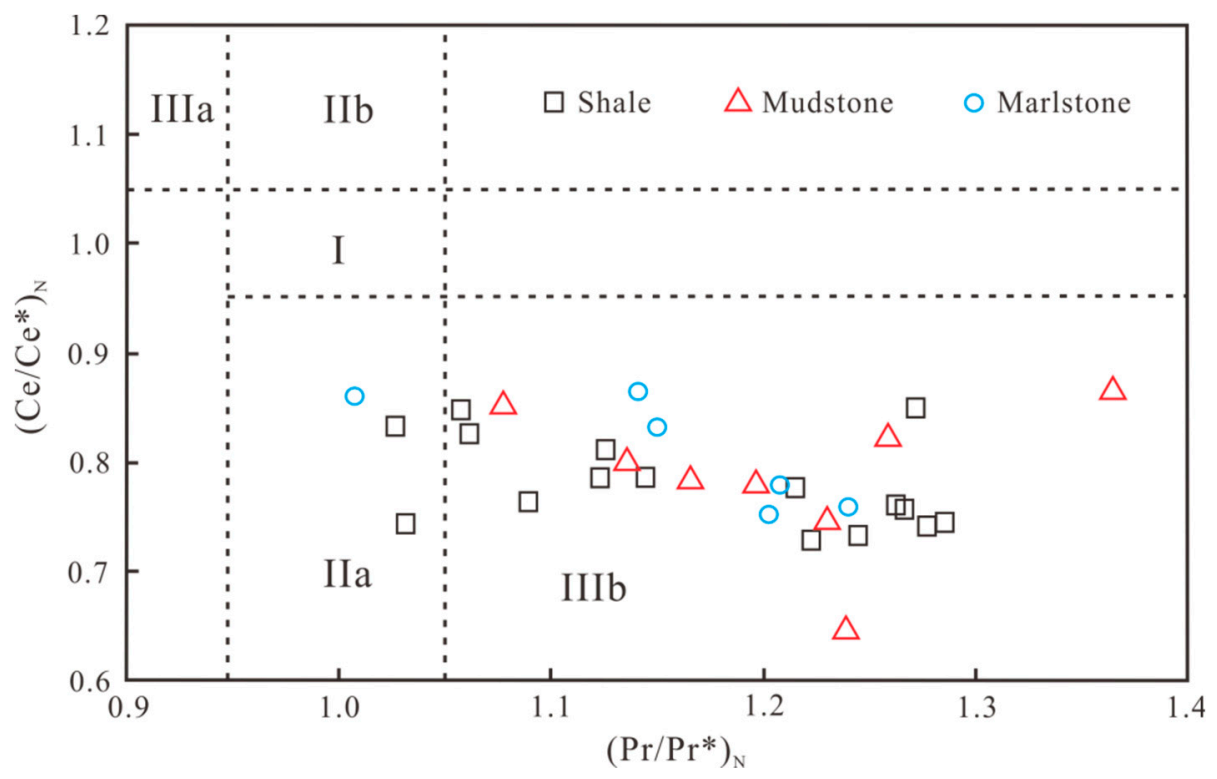

Figure 7. Cross plots demonstrating the relationship between $\mathrm{Pr} / \mathrm{Pr} *$ against $\mathrm{Ce} / \mathrm{Ce}$ * for 30 black rock series samples from Zhenning section. Base map modified from [25]. Notes: (a) Field I: neither La anomaly nor Ce anomaly; (b) Field IIa: positive La anomaly, no Ce anomaly; (c) Field Ilb: negative La anomaly, no Ce anomaly; (d) Field IIIa: positive Ce anomaly; (e) Field IIIb: negative Ce anomaly.

Except for four samples (ZN-14, ZN-15, ZN-28, and ZN-29) that have slightly positive Eu anomalies, the rest of the studied samples have evident negative Eu anomalies $\left(\left(\mathrm{Eu} / \mathrm{Eu}^{*}\right)_{\mathrm{N}}=0.84\right.$; Table 4). The $(\mathrm{La} / \mathrm{Yb})_{\mathrm{N}}$ ratio values have been extensively employed for characterizing shale sedimentary rate $[39,42,43]$. The $(\mathrm{La} / \mathrm{Yb})_{\mathrm{N}}$ ratio values of the target samples range from 0.81 to 1.98 with an average of 1.41 (Table 4), indicating a high sedimentary rate during the black rock series deposition in Southern Guizhou Depression. The high sedimentary rate would lead to a weak REE fractionation, being consistent with the trends of the NASC-normalized REE distribution patterns for three different lithologies.

\subsection{Mineralogical Compositions}

The XRD analyses suggest that the black rock series samples are mainly composed of clay (kaolinite, illite, chlorite, mixed-layer illite-smectite (I/S)), quartz, carbonate (calcite and dolomite), feldspar (K-feldspar, plagioclase), pyrite and anhydrite. All of the computed results are listed in Table 5. Marlstone samples are primarily characterized by high contents of carbonate mineral and relatively low contents of clay mineral and quartz. Specifically, the contents of carbonate mineral vary from $43 \%$ to $52 \%$, with an average of $48 \%$, and these corresponding values of clay mineral and quartz are 
in the range of $20-32 \%$ and $21-28 \%$, with an average of $26 \%$ and $24 \%$, respectively. In contrast, shale and mudstone samples are clay rich ranging from $39 \%$ to $76 \%$ (average of $57 \%$ ), and are relatively quartz poor ranging between $19 \%$ and $58 \%$ (average of $34 \%$ ). Note that clay mineral contents take the absolute advantage and carbonate mineral contents are extremely low in shale and mudstone samples.

Additionally, clay minerals consist of illite (37-76\%), I/S (7-47\%), chlorite (9-38\%) and kaolinite $(0-10 \%)$. Actually, the variations of clay compositions represent the diagenetic transformation process of the clay minerals from kaolinite to illite (I/S is the intermediate product) during the continuous compaction, so the clay minerals assemblage patterns can be employed for mirroring the diagenetic stage to some extent. Specifically, the lower contents of I/S (average of $26 \%$ ) and relatively higher illite contents (average of 55\%) suggest that the black rock series are in the middle diagenetic stage A period [44].

Table 5. The mineralogical compositions of the black rock series samples in this study. (unit: wt $\%$ ).

\begin{tabular}{|c|c|c|c|c|c|c|c|c|c|c|c|c|}
\hline \multirow{2}{*}{ Sample No. } & \multicolumn{8}{|c|}{ Mineral Composition } & \multicolumn{4}{|c|}{ Clay Composition } \\
\hline & Clay & Quartz & KF. & Plag. & Cal. & Dol. & Pyrite & Anhy. & Kao. & Illite & Chlo. & $\mathrm{I} / \mathrm{S}$ \\
\hline $\mathrm{ZN}-1$ & 29 & 21 & 2 & 2 & 33 & 11 & 2 & 0 & 7 & 49 & 12 & 32 \\
\hline $\mathrm{ZN}-2$ & 26 & 28 & 3 & 2 & 35 & 14 & 2 & 0 & 5 & 51 & 14 & 30 \\
\hline $\mathrm{ZN}-3$ & 23 & 24 & 2 & 0 & 37 & 12 & 2 & 0 & 9 & 53 & 13 & 25 \\
\hline $\mathrm{ZN}-4$ & 20 & 24 & 0 & 0 & 39 & 13 & 2 & 2 & 10 & 62 & 10 & 18 \\
\hline $\mathrm{ZN}-5$ & 54 & 42 & 2 & 0 & 0 & 0 & 2 & 0 & 0 & 49 & 14 & 37 \\
\hline $\mathrm{ZN}-6$ & 47 & 48 & 3 & 0 & 0 & 0 & 2 & 0 & 0 & 76 & 17 & 7 \\
\hline $\mathrm{ZN}-7$ & 55 & 40 & 2 & 0 & 0 & 0 & 3 & 0 & 0 & 72 & 12 & 16 \\
\hline $\mathrm{ZN}-8$ & 32 & 23 & 2 & 0 & 35 & 8 & 3 & 0 & 0 & 41 & 21 & 38 \\
\hline ZN-9 & 42 & 56 & 0 & 0 & 0 & 0 & 2 & 0 & 0 & 48 & 15 & 37 \\
\hline $\mathrm{ZN}-10$ & 39 & 58 & 0 & 0 & 0 & 0 & 3 & 0 & 0 & 54 & 26 & 20 \\
\hline $\mathrm{ZN}-11$ & 59 & 38 & 0 & 0 & 0 & 0 & 3 & 0 & 0 & 46 & 24 & 30 \\
\hline $\mathrm{ZN}-12$ & 58 & 40 & 0 & 0 & 0 & 0 & 2 & 0 & 0 & 71 & 16 & 13 \\
\hline $\mathrm{ZN}-13$ & 60 & 35 & 2 & 0 & 0 & 0 & 3 & 0 & 0 & 56 & 10 & 34 \\
\hline $\mathrm{ZN}-14$ & 66 & 25 & 3 & 3 & 0 & 0 & 3 & 0 & 0 & 61 & 16 & 23 \\
\hline ZN-15 & 60 & 33 & 3 & 2 & 0 & 0 & 2 & 0 & 0 & 56 & 17 & 27 \\
\hline $\mathrm{ZN}-16$ & 63 & 31 & 2 & 2 & 0 & 0 & 2 & 0 & 0 & 61 & 14 & 25 \\
\hline $\mathrm{ZN}-17$ & 63 & 29 & 3 & 0 & 0 & 0 & 5 & 0 & 0 & 71 & 14 & 15 \\
\hline $\mathrm{ZN}-18$ & 66 & 26 & 6 & 0 & 0 & 0 & 2 & 0 & 0 & 58 & 19 & 23 \\
\hline $\mathrm{ZN}-19$ & 53 & 44 & 2 & 0 & 0 & 0 & 1 & 0 & 0 & 53 & 21 & 26 \\
\hline $\mathrm{ZN}-20$ & 61 & 35 & 2 & 0 & 0 & 0 & 2 & 0 & 0 & 63 & 16 & 21 \\
\hline $\mathrm{ZN}-21$ & 23 & 25 & 0 & 0 & 36 & 14 & 2 & 0 & 0 & 52 & 23 & 25 \\
\hline $\mathrm{ZN}-22$ & 71 & 25 & 1 & 0 & 1 & 1 & 1 & 0 & 0 & 41 & 38 & 21 \\
\hline $\mathrm{ZN}-23$ & 76 & 19 & 0 & 0 & 2 & 2 & 1 & 0 & 0 & 47 & 35 & 18 \\
\hline $\mathrm{ZN}-24$ & 64 & 23 & 0 & 0 & 6 & 4 & 3 & 0 & 0 & 60 & 9 & 31 \\
\hline $\mathrm{ZN}-25$ & 58 & 23 & 0 & 0 & 12 & 5 & 2 & 0 & 0 & 40 & 13 & 47 \\
\hline $\mathrm{ZN}-26$ & 46 & 32 & 0 & 0 & 18 & 0 & 4 & 0 & 0 & 52 & 14 & 34 \\
\hline $\mathrm{ZN}-27$ & 49 & 27 & 5 & 0 & 13 & 3 & 3 & 0 & 0 & 57 & 15 & 28 \\
\hline $\mathrm{ZN}-28$ & 57 & 26 & 0 & 0 & 10 & 6 & 1 & 0 & 0 & 37 & 16 & 47 \\
\hline ZN-29 & 52 & 31 & 4 & 0 & 9 & 2 & 2 & 0 & 3 & 52 & 24 & 21 \\
\hline $\mathrm{ZN}-30$ & 56 & 25 & 0 & 0 & 8 & 5 & 3 & 3 & 8 & 47 & 24 & 21 \\
\hline
\end{tabular}

Note: KF., K-feldspar; Plag., plagioclase; Cal., calcite; Dol., dolomite; Anhy., anhydrite; Kao., kaolinite; Chlo., chlorite.

\section{Discussion}

\subsection{Weathering Degree}

The chemical compositions in sediments generally stand for their essential mineralogy, a typical characteristic which is, in most cases, easily influenced by the chemical weathering degree [45]. Some elemental cations like $\mathrm{Al}, \mathrm{Mg}, \mathrm{Cs}$, and $\mathrm{Rb}$ are usually reserved in weathering profiles while positive ions with smaller atomic radii, such as $\mathrm{Ca}, \mathrm{Na}$, and $\mathrm{K}$ are easily and rapidly washed away as dissolved states $[46,47]$. The lost proportion of elements is proportional to the weathering degree [48]. 
In order to quantify the weathering degree of lutites, which are mainly composed of silicate minerals of clay and silt size, Nesbitt and Young (1982) [46] and Harnois (1988) [49] put forward Chemical Index of Alteration (CIA) and Chemical Index of Weathering (CIW), repectively.

CIA and CIW are defined as follows:

$$
\begin{gathered}
\mathrm{CIA}=\left[\frac{\mathrm{Al}_{2} \mathrm{O}_{3}}{\mathrm{CaO}^{*}+\mathrm{Al}_{2} \mathrm{O}_{3}+\mathrm{Na}_{2} \mathrm{O}+\mathrm{K}_{2} \mathrm{O}}\right] \times 100 \\
\mathrm{CIW}=\left[\frac{\mathrm{Al}_{2} \mathrm{O}_{3}}{\mathrm{CaO}^{*}+\mathrm{Al}_{2} \mathrm{O}_{3}+\mathrm{Na}_{2} \mathrm{O}}\right] \times 100
\end{gathered}
$$

The unit of oxides in the aforementioned equations is mol, and $\mathrm{CaO}^{*}$ merely refers to the amount of $\mathrm{CaO}$ derived from silicate minerals [46]. Under such a circumstance, it is necessary to make a calibration to the measured $\mathrm{CaO}$ content for the existence of carbonate minerals. In this study, $\mathrm{CaO}$ was primarily calibrated for phosphate using the measured $\mathrm{P}_{2} \mathrm{O}_{5}$ content [50], namely:

$$
\mathrm{CaO}^{*}=\mathrm{CaO}-\mathrm{P}_{2} \mathrm{O}_{5} \times 10 / 3
$$

If the "remaining number of moles" is less than that of $\mathrm{Na}_{2} \mathrm{O}$, the mol of $\mathrm{CaO}$ was regarded as that of $\mathrm{CaO}^{*}$. Otherwise, the mol of $\mathrm{CaO}^{*}$ was supposed to be equivalent to that of $\mathrm{Na}_{2} \mathrm{O}$ [50-53].

In this study, except for marlstone samples, the rest of samples consist mostly of clay minerals and quartz, so the CIA and CIW can be applied to characterizing the weathering degree of shale and mudstone other than marlstone. The calculated CIA and CIW values for shale and mudstone samples from Zhenning section are listed in Table 1 . The shales and mudstones have roughly comparable CIA values ranging from 64.63 to 76.87 , with an average of 73.05. The fact that shales and mudstones are characterized by slightly higher CIA values than PAAS (CIA $=69$, referred from [47]) suggests that they were dominantly from a more weathered source than that for PAAS.

The cross plot of CIA versus $\mathrm{Al} / \mathrm{Na}$ ratios reveals that all shale and mudstone samples, along with PAAS, plot in the middle-weathered field (Figure 8). Interestingly, although there is the concurrence of the shale and mudstone samples with variational $\mathrm{Al} / \mathrm{Na}$ ratios, they display similar CIA values (Figure 8). This phenomenon can be resulted from the diagenetic depletion of sodium ions [46,47]. Additionally, similar to CIA, CIW values range between 75.40 and 89.38 with a mean 82.53 for the shale and mudstone samples from Zhenning section. Both CIA and CIW values reveal that the black rock series might be from a moderately weathered source.

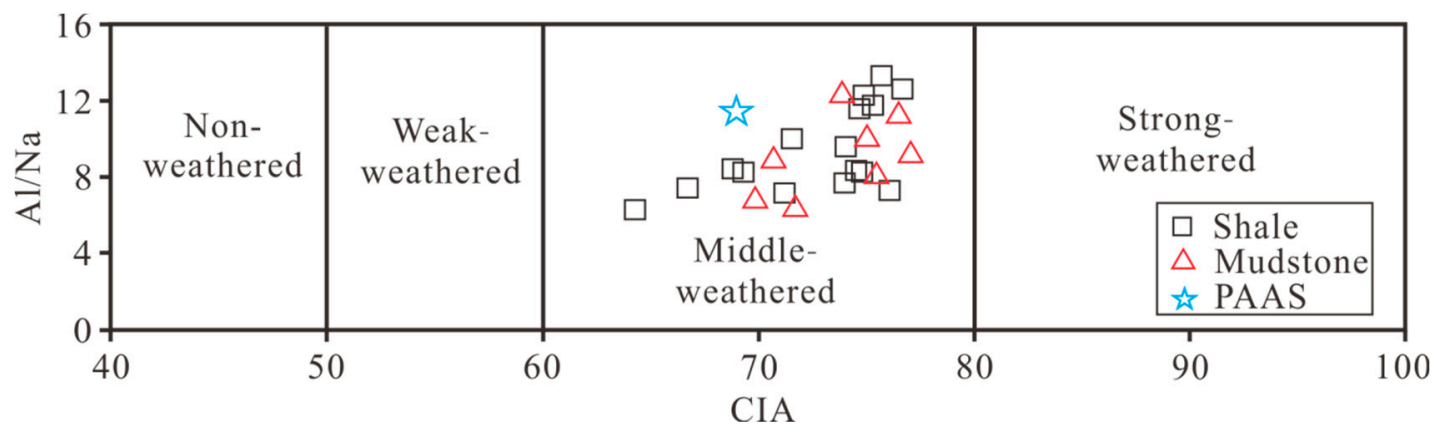

Figure 8. Cross plot of $\mathrm{CIA}$ values versus $\mathrm{Al} / \mathrm{Na}$ ratios demonstrating the weathering degree (the plate after [47]).

\subsection{Provenance}

A comprehensive comparison of the immobile elemental content ratios, for instance $\mathrm{La} / \mathrm{Sc}, \mathrm{La} / \mathrm{Co}$, $\mathrm{Th} / \mathrm{Sc}$, Th/Co, and $\mathrm{Cr} / \mathrm{Th}$ in our investigated samples and their corresponding values of the Upper Continental Crust (UCC), Lower Continental Crust (LCC), and Oceanic Crust (OC) deciphered that the provenance of black rock series in Southern Guizhou Depression is originated from the UCC (Table 6). 
Generally, $(\mathrm{Gd} / \mathrm{Yb})_{\mathrm{N}}$ value is less than 2 in Post-Archean strata while $(\mathrm{Gd} / \mathrm{Yb})_{\mathrm{N}}$ value is more than 2 in Archean strata [32]. In this study, the $(\mathrm{Gd} / \mathrm{Yb})_{\mathrm{N}}$ values of the analyzed samples vary between 0.79 and 1.43 with a mean of 1.05 (Table 4), indicating the provenance of the Datang Formation is from Post-Archean strata.

Furthermore, some non-migratory elements, such as La and Th (indicative of felsic sources) and $\mathrm{Sc}$ (representative of mafic sources) exhibit different chemical properties in felsic and mafic source rocks, so their ratios (e.g., $\mathrm{La} / \mathrm{Sc}, \mathrm{La} / \mathrm{Co}, \mathrm{Th} / \mathrm{Sc}, \mathrm{Th} / \mathrm{Co}$, and $\mathrm{Cr} / \mathrm{Th}$ ) are of value parameters to obtain information about source and composition of the fine-grained rocks $[54,55]$ and are unaffected by the processes of sedimentation [32]. Numerous researchers (e.g., [47,56]) have used these indicators to distinguish between felsic and mafic source rocks. In the present work, these ratios are closer to the values of sediments from felsic rocks relative to those of mafic rocks, thus black rock series from Datang Formation are likely to be felsic (Table 6). The cross plots of some geochemical parameters in Figure 9 can help visualize the source and composition of the target samples. The plot of Th vs. Sc (Figure 9a) suggests that composition of black rock series was dominantly felsic source, implying the single source during the black rock series deposition in Southern Guizhou Depression. Similarly, data for all samples are plotted in the field of felsic volcanic rock and its periphery (Figure 9b). This observation strongly demonstrates that the black rock series were formed by felsic detritus.

In addition, the REEs concentrations can also be used to make inference on the sources of fine-grained rocks $[47,50]$. Previous studies showed that felsic rocks generally exhibit higher LREE/HREE ratios and negative Eu anomalies, while mafic rocks produce low LREE/HREE ratios and no distinct $\mathrm{Eu}$ anomalies (positive or negative) $[32,47,57]$. The Eu anomaly is normally considered as inherited from the parent rocks. Hence, the REEs distribution patterns in fine-grained rocks can help to trace the nature of parent rocks [32]. In view of this regard, constraints on the potential black rock series sources for Datang Formation can be obtained by using the REEs abundances and their distribution patterns. All the investigated samples are characterized by LREE enrichment, negative Eu anomalies and relatively flat HREE NASC-normalized patterns (Figure 6), further suggesting a felsic protolith.

Table 6. Range of several elemental ratios from the black rock series in this study compared with the counterparts in sediments originated from the UCC, LCC, OC, mafic rocks, and felsic rocks.

\begin{tabular}{ccccccc}
\hline $\begin{array}{c}\text { Elemental } \\
\text { Ratios }\end{array}$ & $\begin{array}{c}\text { Range of the Black } \\
\text { Rock Series in This } \\
\text { Study }\end{array}$ & UCC $^{\mathbf{a}}$ & LCC $^{\mathbf{a}}$ & OC $^{\mathbf{b}}$ & $\begin{array}{c}\text { Range of } \\
\text { Sediment from } \\
\text { Mafic Sources }^{\mathbf{b}}\end{array}$ & $\begin{array}{c}\text { Range of } \\
\text { Sediment from }_{\text {Felsic Sources }} \mathbf{b}^{-}\end{array}$ \\
\hline $\mathrm{La} / \mathrm{Sc}$ & $1.76-4.38$ & 2.21 & 0.31 & 0.10 & $0.43-0.76$ & $2.50-16.3$ \\
$\mathrm{La} / \mathrm{Co}$ & $1.09-9.90$ & 1.76 & 0.33 & $/$ & $0.14-0.38$ & $1.80-13.8$ \\
$\mathrm{Th} / \mathrm{Sc}$ & $0.79-1.88$ & 0.79 & 0.06 & 0.94 & $0.05-0.22$ & $0.84-20.5$ \\
$\mathrm{Th} / \mathrm{Co}$ & $0.47-4.25$ & 0.63 & 0.06 & 0.01 & $0.04-1.140$ & $0.67-19.4$ \\
$\mathrm{Cr} / \mathrm{Th}$ & $6.16-34.31$ & 7.76 & 109.5 & $/$ & $25-500$ & $4.00-15.0$ \\
\hline
\end{tabular}

Note: ${ }^{a}$ UCC and LCC data are from [32,50]. ${ }^{\mathrm{b}}$ OC and range of sediment from mafic sources and felsic sources are from [54].

Further, La-Th-Sc and Th-Co-Zr/10 ternary diagrams were performed to decipher the tectonic setting for the Datang Formation [6,58] (Figure 10). The data for the majority of the investigated samples fall within the field of continental island arc in La-Th-Sc ternary diagram (Figure 10a), whereas thay are in the field of active continental margin in $\mathrm{Th}-\mathrm{Co}-\mathrm{Zr} / 10$ ternary diagram (Figure 10b). Therefore, the geochemical characteristics of black rock series demonstrate that the tectonic setting of the Southern Guizhou Depression was continental island arc and active continental margin. This tectonic setting inferred for the provenance of the investigated samples is in accord with the conclusion reported by Yang et al. (1991) [59] based on sedimentological study. 

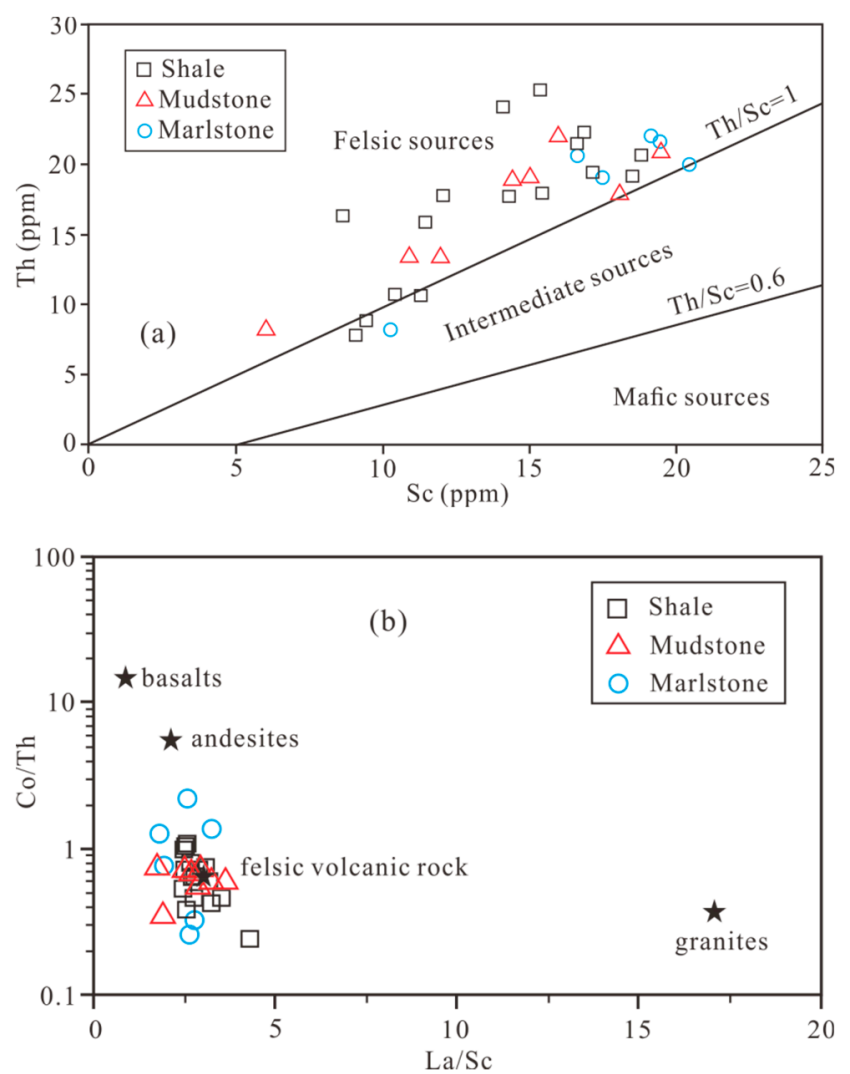

Figure 9. Source and compositional discrimination diagrams of (a) Sc vs. Th and (b) La/Sc vs. Co/Th for the black rock series from Zhenning section. Base map modified from [50].
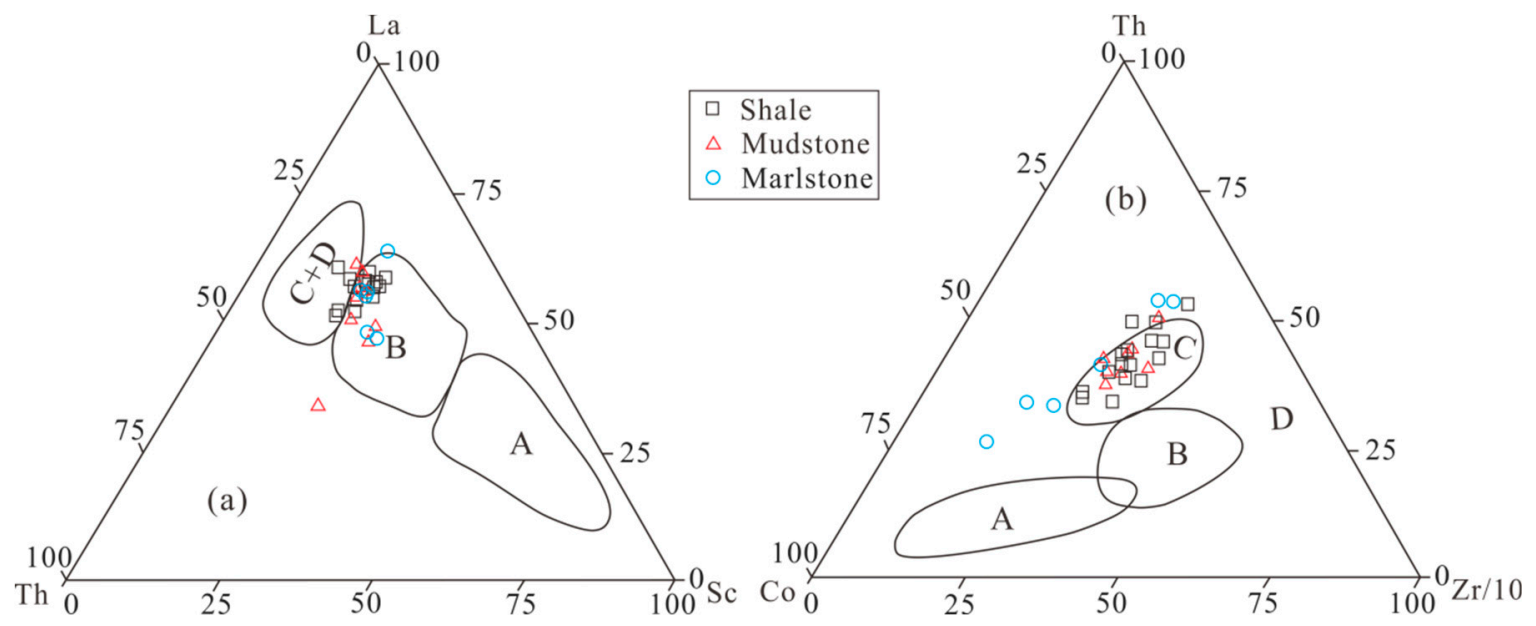

Figure 10. Tectonic setting discrimination diagrams of (a) La-Th-Sc and (b) Th-Co-Zr/10 for the black rock series from Zhenning section (base map after [58]). A: Oceanic island arc; B: Continental island arc; C: Active continental margin; D: Passive continental margin.

\subsection{Paleoredox Conditions}

A number of trace elemens (e.g., $\mathrm{V}, \mathrm{U}, \mathrm{Ni}$ and $\mathrm{Mo}$ ) are sensitive to the redox environments and their ratios (e.g., $\mathrm{U} / \mathrm{Th}, \mathrm{V} / \mathrm{Cr}, \mathrm{Ni} / \mathrm{Co}$ and $\mathrm{V} /(\mathrm{V}+\mathrm{Ni})$ ) have been extensively employed as indicators for paleoredox conditions interpretation $[3,34,60,61]$. Besides, there is a common trend that these ratios reduce with upwelling oxidation degrees in water columns [34]. Previous studies have established the reference standards for $\mathrm{U} / \mathrm{Th}, \mathrm{V} / \mathrm{Cr}, \mathrm{Ni} / \mathrm{Co}$ and $\mathrm{V} /(\mathrm{V}+\mathrm{Ni}$ ) to distinguish anoxic, dysoxic and oxic grades $[36,60,61]$. In the present study, we can see a comparatively good consistence in the analysis of 
redox states applying these standards (Figure 11). These ratios do not show a good correlation with TOC contents (Figure 11), demonstrating oxic and/or dysoxic conditions are unfavorable for organic matter preservation. According to Table 2, all 30 samples from the studied section have the $\mathrm{U} / \mathrm{Th}, \mathrm{V} / \mathrm{Cr}$, $\mathrm{Ni} / \mathrm{Co}$ and $\mathrm{V} /(\mathrm{V}+\mathrm{Ni}$ ) ratio values of $0.15-0.71,0.37-1.28,1.60-16.45$ (the values of $\mathrm{ZN}-3$ and $\mathrm{ZN}-6$ samples more than 10 ) and $0.60-0.88$, with an average of $0.24,1.06,5.69$ and 0.75 , respectively. All these pieces of information indicate that the Lower Carboniferous black rock series were deposited in an oxic/dysoxic environment. This conclusion is also supported by the slightly negative Ce anomalies $\left(\left(\mathrm{Ce} / \mathrm{Ce}^{*}\right)_{\mathrm{N}}=0.91\right.$;Table 4) $[15,39,62]$ and the cross plot of $\mathrm{EF}_{\mathrm{U}}$ vs. $\mathrm{EF}_{\mathrm{Mo}}$ (Figure 12), where all samples plot toward the zone between the oxic end and the dysoxic end of unrestricted marine trend of [63]. More importantly, samples from the relatively lower-TOC marlstones are closer to the oxic end relative to the high-TOC shales/mudstones. This difference of paleoredox conditions is likely to arise from the variations in depositional water depths. Generally, shallower water zones present more oxidizing environments [64]. Therefore, the trace element ratios, slightly negative $\mathrm{Ce}$ anomalies and $\mathrm{EF}_{\mathrm{U}}-\mathrm{EF}_{\mathrm{Mo}}$ covariation together indicate an oxic/dysoxic environment in Southern Guizhou Depression during the early Carboniferous period.
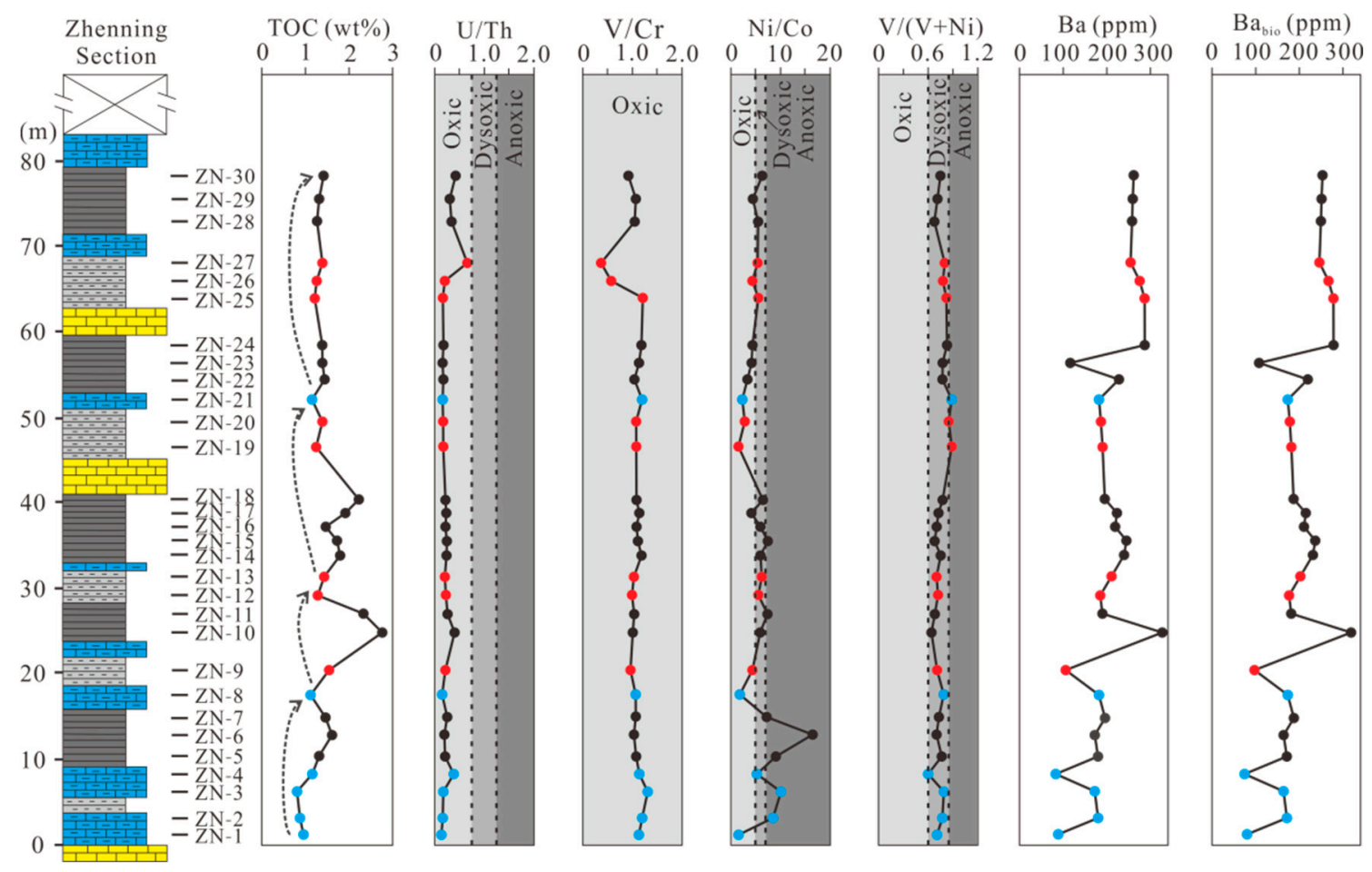

Figure 11. Stratigraphic profiles of Zhenning section for TOC, U/Th, V/Cr, Ni/Co, V/(V + Ni), Ba, and $\mathrm{Ba}_{\text {bio. }}$. Redox thresholds of U/Th $(0.75,1.25), \mathrm{V} / \mathrm{Cr}(2.0)$, and Ni/Co $(5.0,7.0)$ are from [61]; That thresholds of $\mathrm{V} /(\mathrm{V}+\mathrm{Ni})(0.6,0.85)$ are from [60].

\subsection{Paleoclimatic Conditions}

Previous research has suggested that the relative contents and distribution of some major and TEs in fine-grained rocks could infer the paleoclimatic conditions $[6,15,39,65]$. A consensus has been achieved by previous studies $[15,39,66]$ that $\mathrm{Fe}, \mathrm{Mn}, \mathrm{V}, \mathrm{Cr}, \mathrm{Co}$, and $\mathrm{Ni}$ are relatively enriched under moist climatic conditions. On the contrary, the upwelling of water alkalinity owing to evaporation under arid climatic conditions facilitates saline minerals to precipitate, so elements like $\mathrm{Ca}, \mathrm{Mg}, \mathrm{Na}$, $\mathrm{K}, \mathrm{Ba}$, and $\mathrm{Sr}$ are comparatively concentrated. In consideration of the diverse geochemical behaviors of these two groups, their ratios of $\Sigma(\mathrm{Fe}+\mathrm{Mn}+\mathrm{Cr}+\mathrm{Ni}+\mathrm{V}+\mathrm{Co}) / \Sigma(\mathrm{Ca}+\mathrm{Mg}+\mathrm{Sr}+\mathrm{Ba}+\mathrm{K}+\mathrm{Na})$ (named as $\mathrm{C}$-value) are regarded as a climate proxy and have been widely applied to interpreting the paleoclimate [15,39]. Growing numbers of researchers (e.g., $[18,67])$ have concluded that C-values 
of $0-0.2,0.2-0.4,0.4-0.6,0.6-0.8,0.8-1.0$ indicate arid, semiarid, semiarid to semihumid, semihumid, and humid climate respectively.

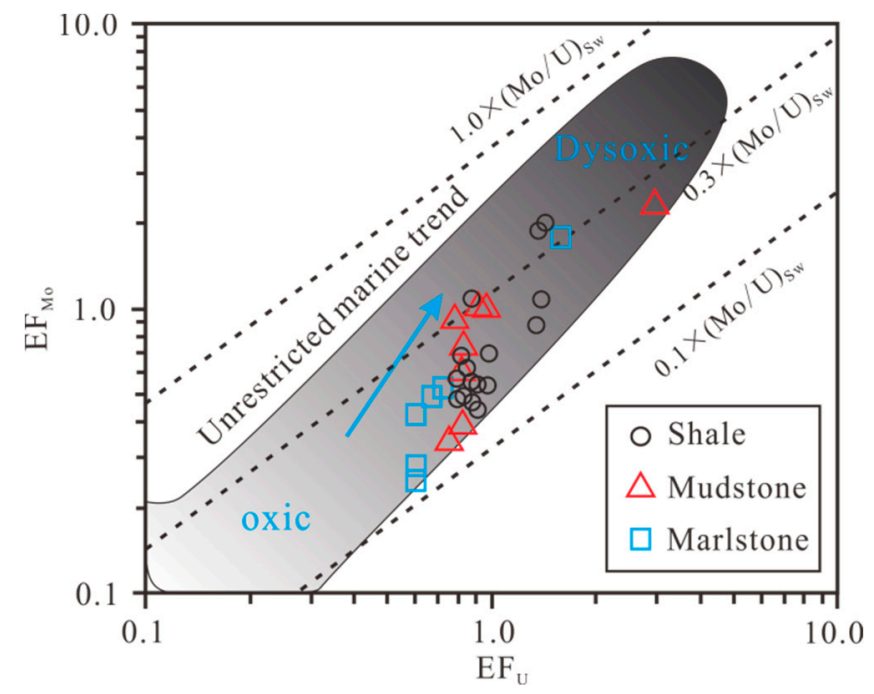

Figure 12. Cross plot of $\mathrm{EF}_{\mathrm{U}} \mathrm{vs}$. $\mathrm{EF}_{\mathrm{Mo}}$, showing that samples from the studied section plot toward the zone between the oxic end and the dysoxic one. Base figure modified from [63]. The diagonal dashed lines represent $\mathrm{Mo} / \mathrm{U}$ molar ratios of the seawater $(\mathrm{Sw})$.

In this study, the C-values for the shale and mudstone samples vary from 0.23 to 0.53 with an average of 0.40 (Table 1), likely mirroring a semiarid climatic condition. However, the corresponding values for the marlstone samples vary between 0.14 and 0.23 with a mean of 0.18 and obviously lower than those of the shale and mudstone samples, indicating an arid climatic condition during the marlstone formation.

In addition, some trace element ratios, such as $\mathrm{Sr} / \mathrm{Cu}$ and $\mathrm{Ga} / \mathrm{Rb}$ ratios, can also be employed for characterizing the paleoclimate $[6,65,66]$. Ga is chiefly enriched in the clay minerals, especially in kaolinite, implying warm and humid climatic conditions $[65,68]$. Rb is closely related with illite, mirroring cold and arid climate [65]. The colder and drier the climate goes, the lower the $\mathrm{Ga} / \mathrm{Rb}$ ratios in sediments become. In general, fine-grained sediments are characterized by low $\mathrm{Sr} / \mathrm{Cu}$ ratios and high $\mathrm{Ga} / \mathrm{Rb}$ ratios in warm and humid climatic conditions [6,15]. The value of $\mathrm{Sr} / \mathrm{Cu}$ ratios between 1.3 and 5.0 can indicate warm-humid environments, whereas the value in excess of 5.0 represents hot-arid climatic conditions $[15,66]$.

Figure 13 displays that the values of $\mathrm{Ga} / \mathrm{Rb}$ and $\mathrm{Sr} / \mathrm{Cu}$ ratios in shale and mudstone samples vary $0.14-0.28$ and $3.38-54.17$, on average of 0.19 and 16.5 , respectively, while the corresponding values in marlstone samples are $0.16-0.19$ and $10.39-31.91$, on average of 0.18 and 16.33 , respectively. On the whole, both indicators reflect that Southern Guizhou Depression was characterized by hot-arid climatic conditions during the early Carboniferous period (Figure 13). However, owing to lack of further division for the climatic condition, such as semiarid and semihumid climate, together with the conclusion inferred from C-values, we surmise that Southern Guizhou Depression was undergoing a hot and arid to semiarid climate during the early Carboniferous period. This is different from the warm paleoclimatic condition of deposition during the early Carboniferous period suggested by Luo et al. (1964) [69] based on sedimentological and paleontological studies. 


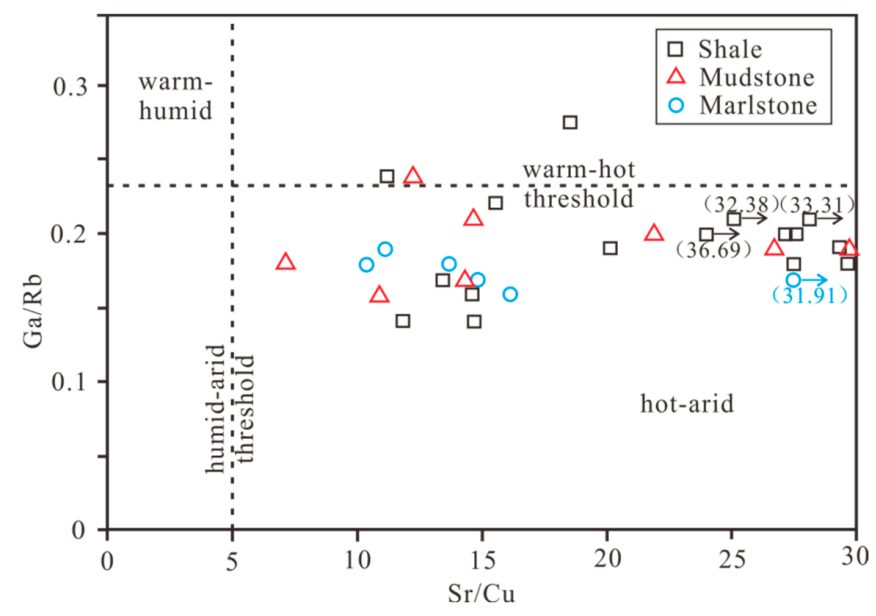

Figure 13. Cross plot of $\mathrm{Sr} / \mathrm{Cu}$ vs. $\mathrm{Ga} / \mathrm{Rb}$ ratios showing paleoclimatic conditions.

\subsection{Paleoproductivity}

Although being decreased during burial process, TOC contents are commonly thought to be associated with the photosynthetic paleoproductivity [12,70]. Therefore, TOC contents can be regarded as a valid indicator for paleoproductivity when combined with other indicators, such as Ba. Based on Figure 3, we can see clearly that TOC variations had a good respond to the stratigraphic cyclicity (except for CCS4), obviously being higher in the middle part of each CCS. This phenomenon can be explained that organic matter is prone to be enriched when regional sea level rises and tends to be decomposed (or depleted) when regional sea level falls.

Biogenic barium $\left(\mathrm{Ba}_{\mathrm{bio}}\right.$ ) has been extensively used in analyzing the fluctuations of palaeoproductivity in marine sediments [71,72]. Determining Ba source is of great significance for applying Ba as a paleoproductivitiy indicator. Except for biogenic sources, there are many other sources probably contributing to Ba accumulation in marine sediments, namely: (1) hydrothermal precipitates; (2) detrital aluminosilicates; and (3) benthic organisms secretion [72]. In this study, no relevant evidence has suggested that Ba originated from hydrothermal precipitates and benthic organisms secretion by far. A quite popular practice for distinguishing between biogenic barium and detrital one is based on the following equation [72]:

$$
\mathrm{Ba}_{\text {bio }}=\mathrm{Ba}_{\text {sample }}-\left(\mathrm{Al} \times \mathrm{Ba} / \mathrm{Al}_{\text {alu }}\right)=\mathrm{Ba}_{\text {sample }}-(\mathrm{Al} \times 0.0075)
$$

The above expression hypothesizes that all $\mathrm{Al}$ in sediments comes from aluminosilicates and that the $\mathrm{Ba} / \mathrm{Al}_{\mathrm{alu}}$ ratio is calculated independently. In general, a Ba/ $\mathrm{Al}_{\text {alu }}$ ratio of 0.0075 is employed for calculating the $\mathrm{Ba}_{\mathrm{bio}}$ content [72]. As presented in Figure 11, the similar variation trend between un-normalized $\mathrm{Ba}$ and $\mathrm{Ba}_{\text {bio }}$ contents confirms that total $\mathrm{Ba}$ contents dominantly comprise $\mathrm{Ba}_{\text {bio }}$. The Ba content is being relatively lower in the CCS1 and then shows an increase trend upwards in the overlying CCS2, CCS3 and CCS4 (Figure 11), being in agreement with the relative sea level rises, during which the high nutrient fluxes could have greatly boosted paleoproductivity. Furthermore, the vertical variations of $\mathrm{Ba}_{\mathrm{bio}}$ contents have a similar trend with TOC fluctuations (Figure 11), demonstrating that paleoproductivity has a significant effect on organic matter accumulation.

\subsection{Organic Matter Enrichment}

In the past few decades, the mechanisms controlling the organic matter accumulation in modern and ancient sediments have been extensively studied [8-11]. A concensus has been reached that organic matter accumulation calls for particularly favorable geological conditions which are generally thought to be connected with three aspects: including (1) non-oxidizing water environment (preservation) [8,9,73]; (2) high primary productivity (organic carbon fluxes) [74-77]; and (3) a proper sedimentary rate $[31,39,43,78]$. 
As mentioned above, the results of this study indicate that the black rock series in Southern Guizhou Depression was deposited in a profoundly unstable and complex paleoenvironmental condition and chiefly experienced moderate weathering, oxic/dysoxic, hot and arid to semiarid climatic conditions. Moreover, the black shale rocks exhibited a high sedimentary rate.

In view of these unstable and complex conditions, the relatively high TOC suggest a relatively moderate-high paleoproductivity [12,70], while oxic/dysoxic and arid to semiarid climatic conditions are usually considered unfavorable for organic matter accumulation and preservation. Additionally, the higher sedimentary rate has vital influence on organic matter preservation. On one hand, the higher sedimentary rate would result in dilution of organic matter $[39,42]$. On the other hand, under oxic/dysoxic conditions, the higher sedimentary rate can shorten the exposure time of organic matter in the degradation region of aerobic bacteria and thus decrease organic matter decomposition [31,79]. Under this circumstance, high paleoproductivity would inevitably play a decisive role in organic matter enrichment. This is strongly supported by the similar vertical variation trends between the paleoproductivity-mirrored $\mathrm{Ba}_{\mathrm{bio}}$ and TOC contents (Figure 11), further illustrating that organic matter enrichment in such a setting is dominantly controlled by the enhanced paleoproductivity.

From the diagram of Figure 3, we could see clearly that TOC variations shared consistent patterns with stratigraphic cyclicity, being in accordance with the relative sea level fluctuations. A consensus has been reached by previous studies $[12,13]$ that sea level rise could have carried the nutritious bottom waters into surface waters, resulting in the photosynthetic phytoplankton blooms and so promoted organic matter generation. Both, enhanced oxygen consumption for microorganisms breathing and organic matter decomposition could have caused oxygen depletion in water column, particularly in bottom water. Furthermore, during the early Carboniferous period, the studied area was deposited in an inter-platform basin, which would remarkably decelerate the water mass ventilation and further favor organic matter preservation, especially under the storm wave base. All of these analyses illustrated that despite the oxygenated state of water column in Southern Guizhou Depression at the time of black rock series deposition, TOC contents are not necessarily lower as observed because paleoproductivity controlled organic matter accumulation.

On the basis of these findings, an enrichment model of organic matter in black rock series during the early Carboniferous period in the studied region was established (Figure 14).

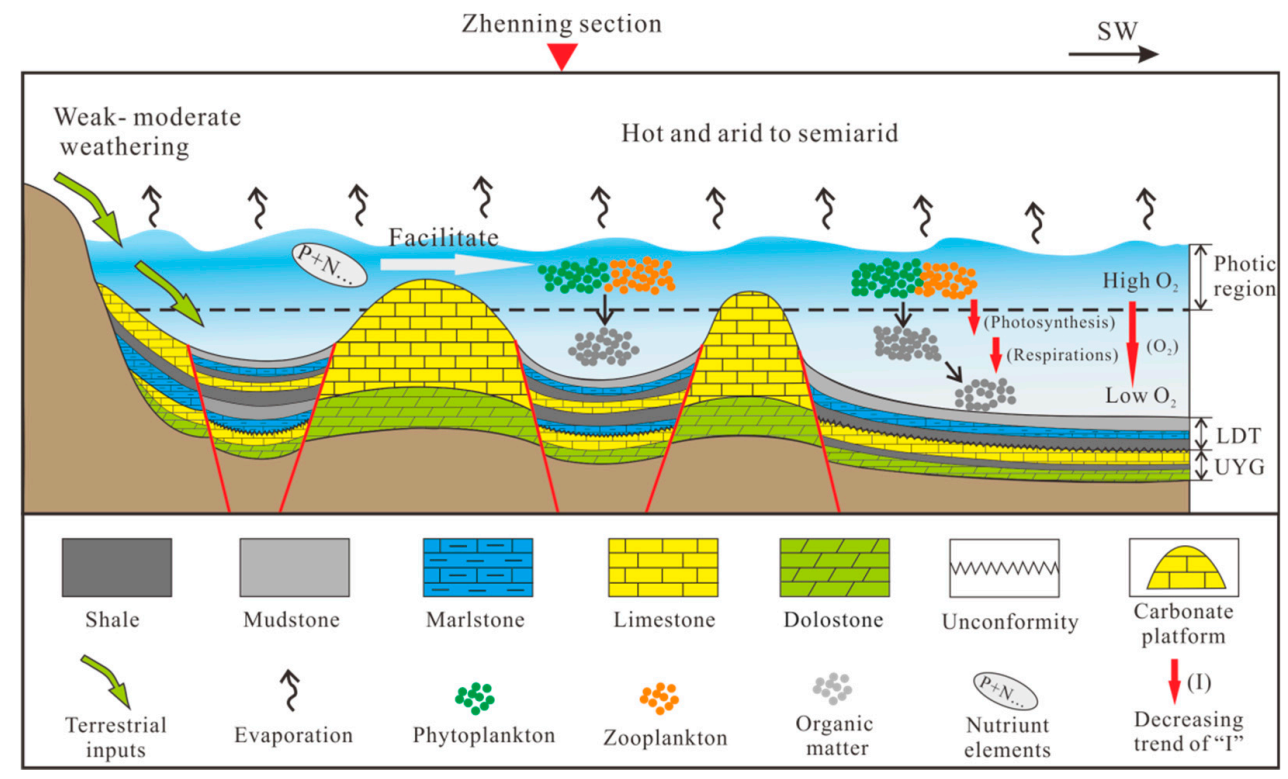

Figure 14. Model illustrating the factors that may have affected organic matter enrichment during the black rock series deposition in Southern Guizhou Depression. UYG and LDT represent the upper part of Yanguan Formation and the lower part of Datang Formation, respectively. 


\section{Conclusions}

This study has provided geochemical data to constrain the depositional conditions and organic matter enrichment in the lower Datang Formation in Southern Guizhou, Southwest China. Based on our data analyses, the following conclusions can be drawn:

(1) The black rock series in Southern Guizhou Depression was deposited in a combination of continental island arc and active continental margin tectonic setting that mainly exhibited moderate weathering, oxic/dysoxic oxygen levels, hot and arid to semiarid climate, and a higher sedimentary rate.

(2) Of the three factors that could affect organic matter enrichment of black rock series strata in an inter-platform basin, including paleoproductivity, redox conditions, and sedimentary rate, the paleoproductivity seems to be the main controlling factor, whereas paleoredox condition of water column and sedimentary rate play relatively less crucial roles.

(3) Despite the oxygenated state of water column in Southern Guizhou Depression at the time of black rock series deposition, TOC contents are not necessarily lower because organic matter accumulation was dominantly controlled by paleoproductivity, which increased due to enhanced nutrient fluxes at a time of regional sea level rise. This, together with the deeper and tranquiller water mass in such an inter-platform basin, would have further accelerated the oxygen depletion via microbial respiration, in turn facilitating organic matter preservation.

Author Contributions: J.D., J.Z. and X.T. designed the experiments, corrected the draft and submitted the paper; J.D., Z.H., S.H., Y.L. and Y.Z. performed the experiments, analyzed the data and participated in writing the drafts; X.L. and T.L. helped in checking the drafts of the paper.

Funding: This research was jointly funded by the National Science and Technology Major Project (Grant No. 2016ZX05034002-001), the National Natural Science Foundation (41802156), the Excellent Supervisor Funded Program of the Ministry of Education (Grant No. 2-9-2017-318), and Open Projects of Key Laboratory of Tectonics and Petroleum Resources, Ministry of Education (TPR-2018-09).

Acknowledgments: The authors thank the three anonymous reviewers for providing constructive suggestions and helpful comments.

Conflicts of Interest: The authors declare no conflict of interest.

\section{References}

1. Bhatia, M.R. Rare earth element geochemistry of Australian Paleozoic graywackes and mudrocks: Provenance and tectonic control. Sediment. Geol. 1985, 45, 97-113. [CrossRef]

2. Schatzel, S.J.; Stewart, B.W. Rare earth element sources and modification in the Lower Kittanning coal bed, Pennsylvania: Implications for the origin of coal mineral matter and rare earth element exposure in underground mines. Int. J. Coal Geol. 2003, 54, 223-251. [CrossRef]

3. Ross, D.J.K.; Bustin, R.M. Investigating the use of sedimentary geochemical proxies for paleoenvironment interpretation of thermally mature organic-rich strata: Examples from the Devonian-Mississippian shales, Western Canadian Sedimentary Basin. Chem. Geol. 2009, 260, 1-19. [CrossRef]

4. Algeo, T.J.; Kuwahara, K.; Sano, H.; Bates, S.; Lyons, T.; Elswick, E.; Linda Hinnov, L.; Moser, J.; Maynard, J.B. Spatial variation in sediment fluxes, redox conditions, and productivity in the Permian-Triassic Panthalassic Ocean. Palaeogeogr. Palaeoclimatol. Palaeoecol. 2011, 308, 65-83. [CrossRef]

5. Blake, J.M.; Peters, S.C.; Johannesson, K.H. Application of REE geochemical signatures for Mesozoic sediment provenance to the Gettysburg Basin, Pennsylvania. Sediment. Geol. 2017, 349, 103-111. [CrossRef]

6. Xie, G.L.; Shen, Y.L.; Liu, S.G.; Hao, W.D. Trace and rare earth element (REE) characteristics of mudstones from Eocene Pinghu Formation and Oligocene Huagang Formation in Xihu Sag, East China Sea Basin: Implications for provenance, depositional conditions and paleoclimate. Mar. Petrol. Geol. 2018, 92, 20-36. [CrossRef]

7. Li, D.L.; Li, R.X.; Xue, T.; Liu, F.T.; Zhao, B.S.; Zhao, D. Characteristic and geological implications of major elements and rare earth elements of Triassic Chang 7 oil shale in Tongchuan city, Southern Ordos Basin (China). Minerals 2018, 8, 157. [CrossRef] 
8. Demaison, G.J.; Moore, G.T. Anoxic environments and oil source bed genesis. AAPG Bull. 1980, 64, 1179-1209. [CrossRef]

9. Arthur, M.A.; Sageman, B.B. Marine black shales: Depositional mechanisms and environments of ancient deposits. Annu. Rev. Earth Planet. Sci. 1994, 22, 499-551. [CrossRef]

10. Wignall, P.B. Black Shale; Oxford University Press: New York, NY, USA, 1994.

11. Murphy, A.E.; Sageman, B.B.; Hollander, D.J.; Lyons, T.W.; Brett, C.E. Black shale deposition and faunal overturn in the Devonian Appalachian Basin: Clastic starvation, seasonal water-column mixing, and efficient biolimiting nutrient recycling. Paleoceanography 2000, 15, 280-291. [CrossRef]

12. Wei, H.Y.; Chen, D.Z.; Wang, J.G.; Yu, H.; Tucker, M.E. Organic accumulation in the lower Chihsia Formation (Middle Permian) of South China: Constraints from pyrite morphology and multiple geochemical proxies. Palaeogeogr. Palaeoclimatol. Palaeoecol. 2012, 353, 73-86. [CrossRef]

13. Wang, S.F.; Zou, C.N.; Dong, D.Z.; Wang, Y.M.; Li, X.J.; Huang, J.L.; Guan, Q.Z. Multiple controls on the paleoenvironment of the Early Cambrian marine black shales in the Sichuan Basin, SW China: Geochemical and organic carbon isotopic evidence. Mar. Petrol. Geol. 2015, 66, 660-672. [CrossRef]

14. Xu, Q.L.; Liu, B.; Ma, Y.S.; Song, X.M.; Wang, Y.J.; Xin, X.K.; Chen, Z.X. Controlling factors and dynamical formation models of lacustrine organic matter accumulation for the Jurassic Da'anzhai Member in the central Sichuan Basin, southwestern China. Mar. Petrol. Geol. 2017, 86, 1391-1405. [CrossRef]

15. Cao, J.; Wu, M.; Chen, Y.; Hu, K.; Bian, L.Z.; Wang, L.G.; Zhang, Y. Trace and rare earth element geochemistry of Jurassic mudstones in the northern Qaidam Basin, northwest China. Chem. Erde-Geochem. 2012, 72, 245-252. [CrossRef]

16. Hu, T.; Pang, X.Q.; Jiang, S.; Wang, Q.F.; Xu, T.W.; Lu, K.; Huang, C.; Chen, Y.Y.; Zheng, X.W. Impact of paleo-salinity, dilution, redox, and paleo-productivity on organic matter enrichment in a saline lacustrine rift basin: A case study of paleogene organic-rich shale in Dongpu Depression, Bohai Bay Basin, Eastern China. Energy Fuels 2018, 32, 5045-5061. [CrossRef]

17. Jiang, D.H. Sedimentary characteristics and evolution of a middle and late Devonian inter-platform basin in Southern Hunan. Sediment. Geol. Teth. Geol. 1990, 6, 21-28. (In Chinese)

18. Mei, M.X.; Ma, Y.S.; Deng, J.; Chu, H.M.; Liu, Z.R.; Zhang, H. Carboniferous to Permian sequence stratigraphic framework of the Yunnan-Guizhou-Guangxi basin and its adjacent areas and global correlation of third-order sea-level change. Chin. Geol. 2005, 32, 13-24. (In Chinese)

19. Tian, J.C.; Kang, J.W.; Lin, X.B.; Zhang, X.; Chen, H. Sequence stratigraphic characteristics and platform basin sedimentary system. J. Southwest Petrol. Univ. 2007, 29, 39-42. (In Chinese)

20. Tian, S.F.; Yang, R.D. Lithofacies and paleogeography evolution and characteristics of shale gas accumulation in Lower Carboniferous, Guizhou, China. J. Chengdu Univ. Technol. 2016, 43, 291-298. (In Chinese)

21. Yang, R.D.; Chen, W.; Zhou, R.X. Characteristics of organic-rich shale and exploration area of shale gas in Guizhou Province. Nat. Gas Geosci. 2012, 23, 340-347. (In Chinese)

22. Zhang, J.C.; Huo, Z.P.; Tang, X.; Liu, Y.; Han, S.B. Shale Gas Geological Characteristics in China; East China University of Technology Press: Shanghai, China, 2016; pp. 26-27. (In Chinese)

23. GGMRB. Guizhou Regional Geology; Geological Publishing House: Beijing, China, 1987; pp. $194-225$. (In Chinese)

24. Yang, R.D.; Yang, C.X.; Wang, C.Y.; Chen, W.Y. The Carboniferous trace fossils in Guizhou: Depression on distribution of Zoophycos throughout geologic history. Guihzou Geol. 1995, 12, 290-297. (In Chinese)

25. Eltom, H.A.; Abdullatif, O.M.; Makkawi, M.H. Rare earth element geochemistry of shallow carbonate outcropping strata in Saudi Arabia: Application for depositional environments prediction. Sediment. Geol. 2017, 348, 51-68. [CrossRef]

26. Cui, J. Sedimentary Response to Teconic Evolution of the Central Guizhou Uplift and Its Adjacent Areas. Ph.D. Dissertation, Central South University, Changsha, China, 2013; pp. 82-88. (In Chinese)

27. Su, H.M.; Yang, R.D.; Cheng, W.; Zhang, Z. Shale gas accumulation characteristics and advantageous area analysis of Lower Carboniferous Dawuba Formation in Southwestern Guizhou. J. Guizhou Univ. 2017, 34, 41-46. (In Chinese)

28. Goldhammer, R.K. Depositional cycles, composite sea-level changes, cycle stacking patterns, and the hierarchy of stratigraphic forcing: Examples from Alpine Triassic platform carbonates. Geol. Soc. Am. Bull. 1990, 102, 535-562. [CrossRef] 
29. Chen, D.; Tucker, M.E. The Frasnian-Famennian mass extinction: Insights from high-resolution sequence stratigraphy and cyclostratigraphy in South China. Palaeogeogr. Palaeoclimatol. Palaeoecol. 2003, 193, 87-111. [CrossRef]

30. Goto, A.; Tatsumi, Y. Quantitative analysis of rock samples by an X-ray fluorescence spectrometer (I). Rigaku J. 1994, 11, 40-59.

31. Cao, J.; Yang, R.F.; Yin, W.; Hu, G.; Bian, L.Z.; Fu, X.G. Mechanism of organic matter accumulation in residual bay environments: The Early Cretaceous Qiangtang Basin, Tibet. Energy Fuel 2018, 32, 1024-1037. [CrossRef]

32. Taylor, S.R.; Mclennan, S.M. The Continental Crust: Its Composition and Evolution; Blackwell Scientific Publications: Hoboken, NJ, USA, 1985; p. 312.

33. Calvert, S.E.; Pedersen, T.F. Geochemistry of Recent oxic and anoxic marine sediments: Implications for the geological record. Mar. Geol. 1993, 113, 67-88. [CrossRef]

34. Rimmer, S.M. Geochemical paleoredox indicators in Devonian-Mississippian black shales, Central Appalachian Basin (USA). Chem. Geol. 2004, 206, 373-391. [CrossRef]

35. Wedepohl, K.H. Environmental influences on the chemical composition of shales and clays. Phys. Chem. Earth 1971, 8, 305-333. [CrossRef]

36. Tribovillard, N.; Algeo, T.J.; Lyons, T.; Riboulleau, A. Trace metals as paleoredox and paleoproductivity proxies: An update. Chem. Geol. 2006, 232, 12-32. [CrossRef]

37. Spears, D.A.; Zheng, Y. Geochemistry and origin of elements in some UK coals. Int. J. Coal Geol. 1999, 38, 161-179. [CrossRef]

38. Fu, X.G.; Wang, J.; Zeng, Y.H.; Tan, F.W.; He, J.L. Geochemistry and origin of rare earth elements (REEs) in the Shengli River oil shale, northern Tibet, China. Chem. Erde-Geochem. 2011, 71, 21-30. [CrossRef]

39. Wang, Z.W.; Fu, X.G.; Feng, X.L.; Song, C.; Wang, D.; Chen, W.B.; Zeng, S.Q. Geochemical features of the black shales from the Wuyu Basin, southern Tibet: Implications for palaeoenvironment and palaeoclimate. Geol. J. 2017, 52, 282-297. [CrossRef]

40. Haskin, L.A.; Wildeman, T.R.; Haskin, M.A. An accurate procedure for the determination of the rare earths by neutron activation. J. Radioanal. Chem. 1968, 1, 337-348. [CrossRef]

41. Bau, M.; Dulski, P. Distribution of yttrium and rare-earth elements in the Penge and Kuruman iron-formations, Transvaal Supergroup, South Africa. Precambrian Res. 1996, 79, 37-55. [CrossRef]

42. Zhang, M.; Liu, Z.; Xu, S.; Sun, P.; Hu, X. Element response to the ancient lake information and its evolution history of argillaceous source rocks in the Lucaogou Formation in Sangonghe area of southern margin of Junggar Basin. J. Earth Sci.-China 2013, 24, 987-996. [CrossRef]

43. Zeng, S.Q.; Wang, J.; Fu, X.G.; Chen, W.B.; Feng, X.L.; Wang, D.; Song, C.Y.; Wang, Z.W. Geochemical characteristics, redox conditions, and organic matter accumulation of marine oil shale from the Changliang Mountain area, northern Tibet, China. Mar. Petrol. Geol. 2015, 64, 203-221. [CrossRef]

44. Yu, B.S.; Li, J.; Zeng, Q.N.; Sun, M.D.; Shi, M. Sedimentary Environment and Diagenesis of Rich Organic Shale; East China University of Technology Press: Shanghai, China, 2016; p. 125. (In Chinese)

45. Nesbitt, H.W.; Young, G.M. Prediction of some weathering trends of plutonic and volcanic rocks based on thermodynamic and kinetic considerations. Geochim. Cosmochim. Acta 1984, 48, 1523-1534. [CrossRef]

46. Nesbitt, H.W.; Young, G.M. Early proterozoic climates and plate motions inferred from major element chemistry of lutites. Nature 1982, 299, 715-717. [CrossRef]

47. Kasanzu, C.; Maboko, M.A.H.; Manya, S. Geochemistry of fine-grained clastic sedimentary rocks of the Neoproterozoic Ikorongo Group, NE Tanzania: Implications for provenance and source rock weathering. Precambrian Res. 2008, 164, 201-213. [CrossRef]

48. Condie, K.C. Chemical composition and evolution of the upper continental crust: Contrasting results from surface samples and shales. Chem. Geol. 1993, 104, 1-37. [CrossRef]

49. Harnois, L. The CIW index: A new chemical index of weathering. Sediment. Geol. 1988, 55, 319-322. [CrossRef]

50. Mclennan, S.M.; Hemming, S.; Mcdaniel, D.K.; Hanson, G.N. Geochemical approaches to sedimentation, provenance, and tectonics. Geol. Soc. Am. 1993, 284, 21-40.

51. Bock, B.; Mclennan, S.M.; Hanson, G.N. Geochemistry and provenance of the Middle Ordovician Austin Glen Member (Normanskill Formation) and the Taconian Orogeny in New England. Sedimentology 2010, 45, 635-655. [CrossRef] 
52. Yan, D.T.; Chen, D.Z.; Wang, Q.C.; Wang, J.G. Large-scale climatic fluctuations in the latest Ordovician on the Yangtze block, south China. Geology 2010, 38, 599-602. [CrossRef]

53. Tang, X.; Zhang, J.C.; Liu, Y.; Yang, C.; Chen, Q.; Dang, W.; Zhao, P.W. Geochemistry of organic matter and elements of black shale during weathering in Northern Guizhou, Southwestern China: Their mobilization and inter-connection. Chem. Erde 2018, 78, 140-151. [CrossRef]

54. Cullers, R.L.; Podkovyrov, V.N. Geochemistry of the Mesoproterozoic Lakhanda shales in southeastern Yakutia, Russia: Implications for mineralogical and provenance control, and recycling. Precambrian Res. 2000, 104, 77-93. [CrossRef]

55. Nowrouzi, Z.; Moussavi-Harami, R.; Mahboubi, A.; Gharaie, M.H.M.; Ghaemi, F. Petrography and geochemistry of Silurian Niur sandstones, Derenjal Mountains, East Central Iran: Implications for tectonic setting, provenance and weathering. Arab. J. Geosci. 2014, 7, 2793-2813. [CrossRef]

56. Mclennan, S.M.; Nance, W.B.; Taylor, S.R. Rare earth element-thorium correlations in sedimentary rocks, and the composition of the continental crust. Geochim. Cosmochim. Acta 1980, 44, 1833-1839. [CrossRef]

57. Roddaz, M.; Viers, J.; Brusset, S.; Baby, P.; Boucayrand, C.; Hérail, G. Controls on weathering and provenance in the Amazonian foreland basin: Insights from major and trace element geochemistry of Neogene Amazonian sediments. Chem. Geol. 2006, 226, 31-65. [CrossRef]

58. Bhatia, M.R.; Crook, K.A.W. Trace element characteristics of graywackes and tectonic setting discrimination of sedimentary basins. Contrib. Mineral. Petrol. 1986, 92, 181-193. [CrossRef]

59. Yang, R.D.; Wu, X.H.; Yan, C.X.; Wang, C.Y. Geochemical character of Carboniferous sediment in Guizhou Province. J. Guizhou Inst. Technol. 1991, 20, 34-41. (In Chinese)

60. Hatch, J.R.; Leventhal, J.S. Relationship between inferred redox potential of the depositional environment and geochemistry of the Upper Pennsylvanian (Missourian) Stark Shale Member of the Dennis Limestone, Wabaunsee County, Kansas, U.S.A. Chem. Geol. 1992, 99, 65-82. [CrossRef]

61. Jones, B.; Manning, D.A.C. Comparison of geochemical indices used for the interpretation of palaeoredox conditions in ancient mudstones. Chem. Geol. 1994, 111, 111-129. [CrossRef]

62. Shields, G.; Stille, P. Diagenetic constraints on the use of cerium anomalies as palaeoseawater redox proxies: An isotopic and REE study of Cambrian phosphorites. Chem. Geol. 2001, 175, 29-48. [CrossRef]

63. Algeo, T.J.; Tribovillard, N. Environmental analysis of paleoceanographic systems based on molybdenum-uranium covariation. Chem. Geol. 2009, 268, 211-225. [CrossRef]

64. Zhou, L.; Algeo, T.J.; Shen, J.; Hu, Z.; Gong, H.; Xie, S.C.; Huang, J.H.; Gao, S. Changes in marine productivity and redox conditions during the Late Ordovician Hirnantian glaciation. Palaeogeogr. Palaeoclimatol. Palaeoecol. 2015, 420, 223-234. [CrossRef]

65. Roy, D.K.; Roser, B.P. Climatic control on the composition of Carboniferous-Permian Gondwana sediments, Khalaspir basin, Bangladesh. Gondwana Res. 2013, 23, 1163-1171. [CrossRef]

66. Yandoka, B.M.S.; Wan, H.A.; Abubakar, M.B.; Hakimi, M.H.; Adegoke, A.K. Geochemical characterisation of Early Cretaceous lacustrine sediments of Bima Formation, Yola Sub-basin, Northern Benue Trough, NE Nigeria: Organic matter input, preservation, paleoenvironment and palaeoclimatic conditions. Mar. Petrol. Geol. 2015, 61, 82-94. [CrossRef]

67. Yang, W.Q.; Jiang, Y.L.; Yong, W. Study on shale facies sedimentary environment of lower Es3-upper Es4 in Dongying sag. J. China Univ. Petrol. 2015, 39, 19-26. (In Chinese)

68. Beckmann, B.; Flögel, S.; Hofmann, P.; Schulz, M.; Wagner, T. Orbital forcing of Cretaceous river discharge in tropical Africa and ocean response. Nature 2005, 437, 241-244. [CrossRef] [PubMed]

69. Luo, S.W.; Fu, K. An outline map of lithofacies paleogeography in Guizhou Province. J. Guizhou Inst. Technol. 1964, 0, 24-43. (In Chinese)

70. Nara, F.; Tani, Y.; Soma, Y.; Soma, M.; Naraoka, H.; Watanabe, T.; Horiuchi, K.; Kawai, T.; Oda, T.; Nakamura, T. Response of phytoplankton productivity to climate change recorded by sedimentary photosynthetic pigments in Lake Hovsgol (Mongolia) for the last 23,000 years. Quat. Int. 2005, 136, 71-81. [CrossRef]

71. Francois, R.; Honjo, S.; Manganini, S.J.; Ravizza, G.E. Biogenic barium fluxes to the deep sea: Implications for paleoproductivity reconstruction. Glob. Biogeochem. Cycles 1995, 9, 289-303. [CrossRef]

72. Dymond, J.; Suess, E.; Lyle, M. Barium in deep-sea sediment: A geochemical proxy for paleoproductivity. Paleoceanography 1992, 7, 163-181. [CrossRef] 
73. Mort, H.; Jacquat, O.; Adatte, T.; Steinmann, P.; Föllmi, K.; Matera, V.; Berner, Z.; Stüben, D. The Cenomanian/Turonian anoxic event at the Bonarelli Level in Italy and Spain: Enhanced productivity and/or better preservation? Cretac. Res. 2007, 28, 597-612. [CrossRef]

74. Pedersen, T.F.; Calvert, S.E. Anoxia vs. productivity: What controls the formation of organic-carbon-rich sediments and sedimentary rocks? AAPG Bull. 1990, 74, 454-466.

75. Caplan, M.L.; Bustin, R.M. Palaeoceanographic controls on geochemical characteristics of organic-rich Exshaw mudrocks: Role of enhanced primary production. Org. Geochem. 1999, 30, 161-188. [CrossRef]

76. Sageman, B.B.; Murphy, A.E.; Werne, J.P.; Ver Straeten, C.A.; Hollander, D.J.; Lyons, T.W. A tale of shales: The relative roles of production, decomposition, and dilution in the accumulation of organic-rich strata, Middle-Upper Devonian, Appalachian basin. Chem. Geol. 2003, 195, 229-273. [CrossRef]

77. Gallego-Torres, D.; Martínez-Ruiz, F.; Paytan, A.; Jiménez-Espejo, F.; Ortega-Huertas, M. Pliocene-Holocene evolution of depositional conditions in the eastern Mediterranean: Role of anoxia vs. productivity at time of sapropel deposition. Palaeogeogr. Palaeoclimatol. Palaeoecol. 2007, 246, 424-439. [CrossRef]

78. Tyson, R.V. The "productivity versus preservation" controversy: Cause, flaws, and resolution. Soc. Sediment. Geol. 2005, 82, 17-33.

79. Ibach, L.E.J. Relationship between sedimentation rate and total organic carbon content in ancient marine sediments. AAPG Bull. 1982, 66, 170-183.

(C) 2018 by the authors. Licensee MDPI, Basel, Switzerland. This article is an open access article distributed under the terms and conditions of the Creative Commons Attribution (CC BY) license (http:/ / creativecommons.org/licenses/by/4.0/). 